\title{
Models for strongly-nonlinear evolution of long internal waves in a two-layer stratification
}

\author{
T. Sakai and L. G. Redekopp \\ Department of Aerospace \& Mechanical Engineering, University of Southern California, Los Angeles, CA 90089-1191, USA
}

Received: 1 June 2006 - Revised: 19 January 2007 - Accepted: 26 January 2007 - Published: 30 January 2007

\begin{abstract}
Models describing the evolution of long internal waves are proposed that are based on different polynomial approximations of the exact expression for the phase speed of uni-directional, fully-nonlinear, infinitely-long waves in the two-layer model of a density stratified environment. It is argued that a quartic KdV model, one that employs a cubic polynomial fit of the separately-derived, nonlinear relation for the phase speed, is capable of describing the evolution of strongly-nonlinear waves with a high degree of fidelity. The marginal gains obtained by generating higher-order, weaklynonlinear extensions to describe strongly-nonlinear evolution are clearly demonstrated, and the limitations of the quite widely-used quadratic-cubic KdV evolution model obtained via a second-order, weakly-nonlinear analysis are assessed. Data are presented allowing a discriminating comparison of evolution characteristics as a function of wave amplitude and environmental parameters for several evolution models.
\end{abstract}

\section{Preliminary considerations}

The wide-spread appearance of packets of long internal waves in the shallow, stratified waters of the coastal ocean and lakes is firmly established (Osborne and Burch, 1980; Apel et al., 1985; Scotti and Pineda, 2004; Ostrovsky and Stepanyants, 1989; Stanton and Ostrovsky, 1998; Antenucci et al., 2000; Duda et al., 2004; Helfrich and Melville, 2006). These long-wave packets in many contexts are decidedly nonlinear, containing waves with amplitudes that are equal to and greater in magnitude than the controlling length scale of the problem, which is typically the scale of the uppermixed layer depth. Furthermore, these long-wave packets are known to stimulate strong benthic dissipation and mixing, having a determining influence on the decay of internal

Correspondence to: T. Sakai

(tsakai@usc.edu) tidal energy and the resuspension and transport of sedimentary material (Bogucki et al., 1997; Bogucki and Redekopp, 1999; Stastna and Lamb, 2002; Bogucki et al., 2005). Hence, there is increasing interest in the development of models that can yield quantitative, evolutionary descriptions of such wave packets in a wide range of environments. The present work is directed toward proposing such a model, and assessing its potential (along with that of various alternate models) to describe reliable descriptions of packets possessing large amplitude internal waves.

Aspects of the propagation of nonlinear internal waves in the long-wave limit are examined in the case of a two-layer model under the Boussinesq approximation. Specifically, uni-directional propagation of a plane wave is considered in the idealized environmental model of a two-layer density stratification. This idealized case represents a convenient and quantitatively relevant model for the lowest internal wave mode in realistic stratifications when the water column possesses a single, prominent, thermoclinic layer whose depth is at most a modest fraction of the fluid depth. In what follows, the characteristics of the environmental model are defined in terms of the undisturbed, upper (lower) layer depths $h_{1}\left(h_{2}\right)$ and respective fluid densities $\rho_{1}\left(\rho_{2}\right)$, and the interface displacement is denoted by the function $\zeta(x, t)$. Within the Boussinesq approximation the layer densities enter only through the reduced gravity $\tilde{g}=g\left(\rho_{2}-\rho_{1}\right) / \rho_{1}$.

It is well established that the weakly-nonlinear evolution of an interfacial gravity wave in the limit of two shallow layers is described by the Korteweg-deVries (KdV) equation (Grimshaw, 2002; Helfrich and Melville, 2006). It is also quite well established that theoretical correspondence with experiments and field data is significantly enhanced when an extended (i.e., a quadratic-cubic) KdV equation is employed (cf., Grimshaw, 2002; Holloway and Pelinovsky, 2002; Helfrich and Melville, 2006). That is, the preferred evolutionary model, when written in dimensional variables, has the extended form

Published by Copernicus GmbH on behalf of the European Geosciences Union and the American Geophysical Union. 
$\zeta_{t}+c_{0}\left(1-\alpha_{1} \zeta-\alpha_{2} \zeta^{2}\right) \zeta_{x}+\beta_{0} c_{0} \zeta_{x x x}=0$

In the terminology adopted here this equation is referred to as $\mathrm{KdV} 2$ when $\alpha_{2} \neq 0$, and as $\mathrm{KdV} 1$ when $\alpha_{2}=0 . \mathrm{KdV} 1$ is the familiar and widely-studied equation where the nonlinearity derives solely from the leading-order, nonlinear correction to the linear long-wave phase speed $c_{0}$. KdV2 represents the case where both the first-order and second-order dependencies (i.e., linear and quadratic terms in an asymptotic approximation for small amplitudes) of the long-wave phase speed on wave amplitude are incorporated into the evolution model. The coefficients in this equation have the following representations in terms of the layer depths for the two-layer environmental model:

$$
\begin{aligned}
& c_{0}^{2}=\frac{\tilde{g} h_{1} h_{2}}{h_{1}+h_{2}}=\frac{1}{2} \tilde{g} h\left(1-\epsilon^{2}\right) ; \\
& \alpha_{1}=\frac{3}{2} \frac{h_{2}-h_{1}}{h_{1} h_{2}}=\frac{3 \epsilon}{h\left(1-\epsilon^{2}\right)} \equiv \frac{\tilde{\alpha}_{1}}{h} ; \\
& \alpha_{2}=\frac{3}{8} \frac{\left(h_{2}-h_{1}\right)^{2}+8 h_{1} h_{2}}{\left(h_{1} h_{2}\right)^{2}}=\frac{3}{2} \frac{2-\epsilon^{2}}{h^{2}\left(1-\epsilon^{2}\right)^{2}} \equiv \frac{\tilde{\alpha}_{2}}{h^{2}} ; \\
& \beta_{0}=\frac{1}{6} h_{1} h_{2}=\frac{1}{6} h^{2}\left(1-\epsilon^{2}\right) .
\end{aligned}
$$

These coefficients are given in two different forms, the latter obtained by using the alternate representations $h_{1}=h(1-\epsilon)$, $h_{2}=h(1+\epsilon)$ for the layer depths with $h$ equal to one-half the fluid column depth and $\epsilon=\left(h_{2}-h_{1}\right) / 2 h$.

The fact that the coefficients of various terms in the evolution Eq. (1) are available in terms of analytical expressions that are easily calculated makes the two-layer model attractive for first-order estimates of wave properties. Nevertheless, a nagging question relating to any asymptotic approximation is the quantitative utility of representing a nonlinear function in terms of only the leading terms of its asymptotic expansion. This has been a persistent concern regarding the application of KdV theory in contexts where there is a compelling interest in assessing or comparing physical characteristics at a quantitative level (e.g., front propagation speeds, wave profile shapes, local wave-induced velocity shear and mixing, wave-induced benthic stimulation, vertical and horizontal particle transport by a solitary wave or long-wave packet, etc.).

\section{The case of equal layer depths}

The first issue to be addressed is the evolution of the interfacial wave in the limiting case when the layer depths are nearly equal, and when the wave amplitude becomes large. When $\epsilon=0$ one observes from Eqs. (3) and (4) that $\alpha_{1}=0$ and $\alpha_{2}=3 / h^{2}$. In this limiting situation we have a special case of KdV2 and, as noted originally by Djordjevic and Redekopp (1978), both $\alpha_{2}>0$ and $\beta_{0}>0$ so that nonlinear steepening augments the effect of linear dispersion and no equilibrium solutions (e.g., solitary waves) are possible. Also, the evolution equation has the invariance $\zeta \rightarrow-\zeta$ and a waveform of either polarity is possible. This is in contrast to the leading order case for disparate depths (i.e., when $\left|\alpha_{1}\right| \gg\left|\alpha_{2} \zeta\right|$ and the evolution is essentially described by $\mathrm{KdV} 1$ ). Equilibrium solutions of KdV1 are possible when $\alpha_{1} \zeta$ and $\beta_{0}$ have opposite signs, a condition implying that the wave polarity is always such that the interface displacement is a wave of elevation with respect to the shallowest layer.

The key point relating to this first issue is the sign (and magnitude) of the higher-order terms in Eq. (1) when $\epsilon$ tends toward zero, either from positive or negative values. That is, although $\alpha_{1}$ tends to zero with $\epsilon$, what are the sign and magnitude of $\alpha_{3}$, etc., for small values of $|\epsilon|$. The issue is readily addressed through use of a recent result by Slunyaev et al. (2003) where, based on earlier work by Ostrovsky and Grue (2003), an analytic expression for the fully-nonlinear, longwave propagation speed for a wave moving in one direction was derived (see Eq. 45 in Ostrovsky and Stepanyants, 2005):

$$
\begin{aligned}
& \tilde{c}_{E}=\frac{c_{E}}{c_{0}}=1+3 \frac{h_{2}-h_{1}}{\left(h_{1}+h_{2}\right)^{2}}\left(h_{2}-h_{1}+2 \zeta\right) \times \\
&\left\{\sqrt{\frac{\left(h_{1}-\zeta\right)\left(h_{2}+\zeta\right)}{h_{1} h_{2}}-\frac{h_{2}-h_{1}+2 \zeta}{h_{2}-h_{1}}}\right\} \\
&=1-3(\epsilon+\tilde{\zeta})^{2}+3 \epsilon(\epsilon+\tilde{\zeta}) \sqrt{\frac{1-(\epsilon+\tilde{\zeta})^{2}}{1-\epsilon^{2}}} .
\end{aligned}
$$

Dimensionless variables $\left(\tilde{c}_{E}, \tilde{\zeta}\right)=\left(c / c_{0}, \zeta / h\right)$ are employed in the second representation of the expression, termed the "exact" value and denoted by $\tilde{c}_{E}$. It is readily apparent, and quite important to note, that Eq. (6) possesses the symmetry property $\tilde{c}_{E}(\tilde{\zeta} ; \epsilon)=\tilde{c}_{E}(-\tilde{\zeta} ;-\epsilon)$.

The dependence of the phase speed $\tilde{c}_{E}$ on wave amplitude is displayed in Fig. 1 for several ratios of the layer depths (i.e., values of $\epsilon$ ). Results are shown by solid lines in the figure and correspond to waves with physically relevant polarities; that is, waves of depression $(\tilde{\zeta}<0)$ for $\epsilon>0\left(h_{1}<h_{2}\right)$ and waves of elevation $(\tilde{\zeta}>0)$ for $\epsilon<0\left(h_{1}>h_{2}\right)$. One observes immediately that the phase speed is not a monotonic function of the wave amplitude. The negative sign before the $\alpha_{2}$ coefficient causes the long-wave phase speed to diminish as the wave amplitude increases. It is only in the particular case of equal layer depths that the phase speed is a monotonic function of the amplitude, diminishing continuously from its maximum value of unity (i.e., the linear value) as the amplitude increases.

To aid the reader in relating values of the parameter $\epsilon$ to physical dimensions, some representative values for the different ratios of the layer depths are given in Table 1. Included in Table 1 are corresponding values of $\tilde{\zeta}_{1}=\zeta / h_{1}=\tilde{\zeta} /(1-\epsilon)$ to provide a direct conversion of the dimensionless wave amplitude in terms of the upper-layer depth (the controlling length scale for long waves when $\epsilon<0$ ). Further, values of $\tilde{c}_{E m}$, the 


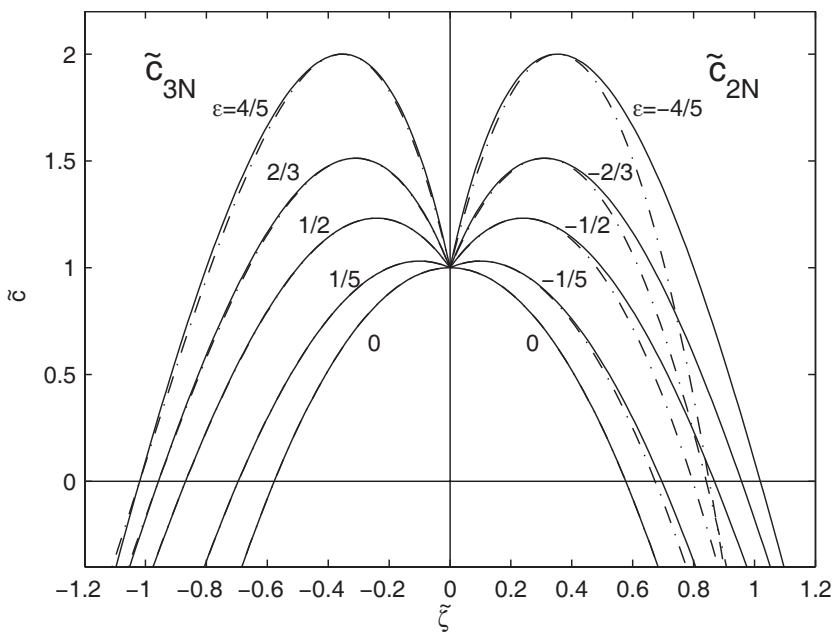

Fig. 1. The exact, fully nonlinear long-wave phase speed as a function of wave amplitude for various layer-depth ratios, including comparison with two polynomial fits $\tilde{c}_{2 \mathrm{~N}}$ and $\tilde{c}_{3 \mathrm{~N}}$.

maximum value of $\tilde{c}_{E}$, and the corresponding wave amplitude $\tilde{\zeta}_{m}$ where the phase speed maximum occurs, are also given in Table 1. Expressions for these relations can be derived from the expression in Eq. (6) and their analytical forms are presented subsequently.

With Eq. (6) in hand, it is straightforward to develop the weakly-nonlinear expansion of the phase speed to orders beyond the quadratic term (i.e., the $\alpha_{2}$ term) that appears in Eq. (1) and is defined in Eq. (4). The expansion of Eq. (6) to higher orders yields the extended expression

$\tilde{c}_{E}=1-\alpha_{1} \zeta-\alpha_{2} \zeta^{2}-\alpha_{3} \zeta^{3}-\alpha_{4} \zeta^{4}-\cdots$

We draw particular attention to the expressions for the following higher-order terms:

$$
\begin{aligned}
\alpha_{3} & =\frac{3}{16} \frac{\left(h_{2}-h_{1}\right)\left(h_{1}+h_{2}\right)^{2}}{\left(h_{1} h_{2}\right)^{3}}=\frac{3}{2} \frac{\epsilon}{h^{3}\left(1-\epsilon^{2}\right)^{3}} \equiv \frac{\tilde{\alpha}_{3}}{h^{3}} ; \\
\alpha_{4} & =\frac{15}{128} \frac{\left(h_{2}-h_{1}\right)^{2}\left(h_{1}+h_{2}\right)^{4}}{\left(h_{1} h_{2}\right)^{4}}=\frac{15}{8} \frac{\epsilon^{2}}{h^{4}\left(1-\epsilon^{2}\right)^{4}} \\
& \equiv \tilde{\alpha}_{4} / h^{4} .
\end{aligned}
$$

One notes that $\tilde{\alpha}_{1}$ and $\tilde{\alpha}_{3}$ (and further $\tilde{\alpha}_{i}$ for odd $i$ ) always carry the same sign, being proportional to $\epsilon=\left(h_{2}-h_{1}\right) / 2 h$. However, and most significantly, all coefficients $\tilde{\alpha}_{i}$ vanish when $h_{2}=h_{1}$ for $i \geq 3$. Consequently, and as pointed out in the note by Slunyaev et al. (2003), the expansion for the nonlinear phase speed for long-wave propagation terminates at the $\tilde{\alpha}_{2}$ term when the layer depths are equal, and the fullynonlinear phase speed when $h_{2}=h_{1}$ is given exactly by the simple polynomial expression

$\tilde{c}_{E}=1-3 \tilde{\zeta}^{2}$.

Stated in another way, the well-established coefficient for the cubic KdV equation (Eq. 1 with $\alpha_{1}=0$ ) contains already the
Table 1. Environmental parameters and corresponding wave properties for a range of depth ratios.

\begin{tabular}{lccccc}
\hline$\epsilon$ & $h_{2} / h_{1}$ & $\tilde{\zeta}_{1}$ & $\tilde{\zeta}_{c}$ & $\tilde{\zeta}_{\max }$ & $\tilde{c}_{E \max }$ \\
\hline 0 & 1 & $\tilde{\zeta}$ & $-1 / \sqrt{3}$ & 0.000 & 1.000 \\
$1 / 5$ & $3 / 2$ & $5 \tilde{\zeta} / 4$ & -0.696 & -0.100 & 1.031 \\
$1 / 3$ & 2 & $3 \tilde{\zeta} / 2$ & -0.773 & -0.164 & 1.091 \\
$1 / 2$ & 3 & $2 \tilde{\zeta}$ & -0.868 & -0.241 & 1.232 \\
$3 / 5$ & 4 & $5 \tilde{\zeta} / 2$ & -0.923 & -0.284 & 1.375 \\
$2 / 3$ & 5 & $3 \tilde{\zeta}$ & -0.957 & -0.310 & 1.513 \\
$3 / 4$ & 7 & $4 \tilde{\zeta}$ & -0.998 & -0.339 & 1.768 \\
$4 / 5$ & 9 & $5 \tilde{\zeta}$ & -1.019 & -0.353 & 2.000 \\
$5 / 6$ & 11 & $6 \tilde{\zeta}$ & -1.032 & -0.361 & 2.214 \\
$6 / 7$ & 13 & $7 \tilde{\zeta}$ & -1.040 & -0.365 & 2.412 \\
$7 / 8$ & 15 & $8 \tilde{\zeta}$ & -1.046 & -0.367 & 2.598 \\
$8 / 9$ & 17 & $9 \tilde{\zeta}$ & -1.050 & -0.368 & 2.774 \\
$9 / 10$ & 19 & $10 \tilde{\zeta}$ & -1.052 & -0.369 & 2.941 \\
\hline
\end{tabular}

exact nonlinear steepening when the layer depths are equal. Furthermore, and as seen in Fig. 1 and noted in Table 1, we find the surprising result that the nonlinear propagation speed diminishes to zero for wave amplitudes of either polarity approaching the limiting value $\left|\tilde{\zeta}_{c}\right|=1 / \sqrt{3}$. This consequence of waves becoming stagnant as the wave amplitude increases was observed in simulations for shoaling of winddriven, long internal waves in a lake ${ }^{1}$, and was an initiatial motivation for the study presented here. The critical values $\tilde{\zeta}_{c}$ (i.e., amplitudes where $\tilde{c}_{E}=0$ ) for other depth ratios are included in Table 1.

\section{Strongly nonlinear long waves}

The second and principal issue addressed pertains to the range of validity of the weakly nonlinear representation of the phase speed and, consequently, the specification of the appropriate "extended" evolution equation for depth ratios that are not near unity. When considering numerical simulations of lowest-mode, long internal wave motion along a single characteristic direction in shallow waters, the exact nonlinear expression $c_{E}(\zeta)$ for the long-wave phase speed given in Eq. (6) can be inserted in place of the quadratic approximation appearing in Eq. (1). However, it is of some interest to have a polynomial approximation for $c_{E}(\zeta)$ in order to obtain an evolution equation that potentially yields analytical (i.e., parameterized) expressions for equilibrium solutions, a hierarchy of conserved densities, and yet possesses the capacity

\footnotetext{
${ }^{1}$ Unpublished report by T. Sakai and L. G. Redekopp.
} 
(a)

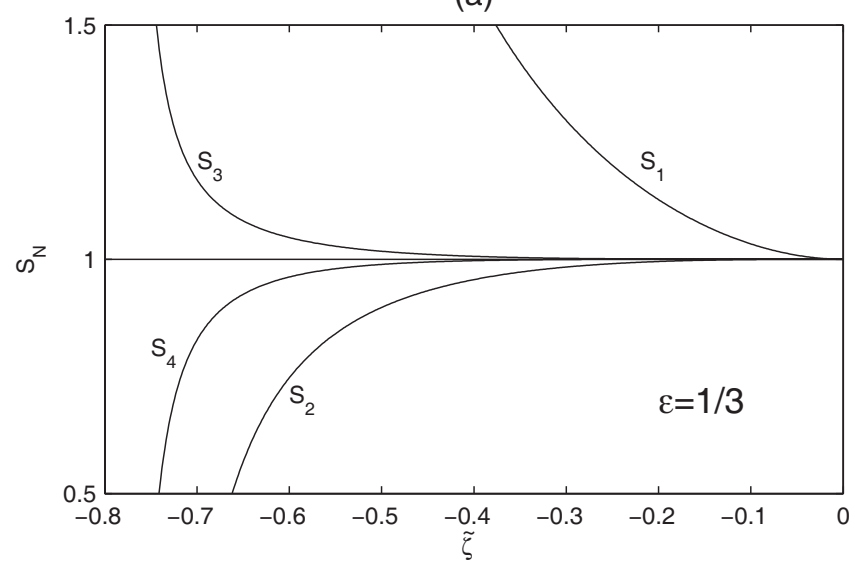

(b)

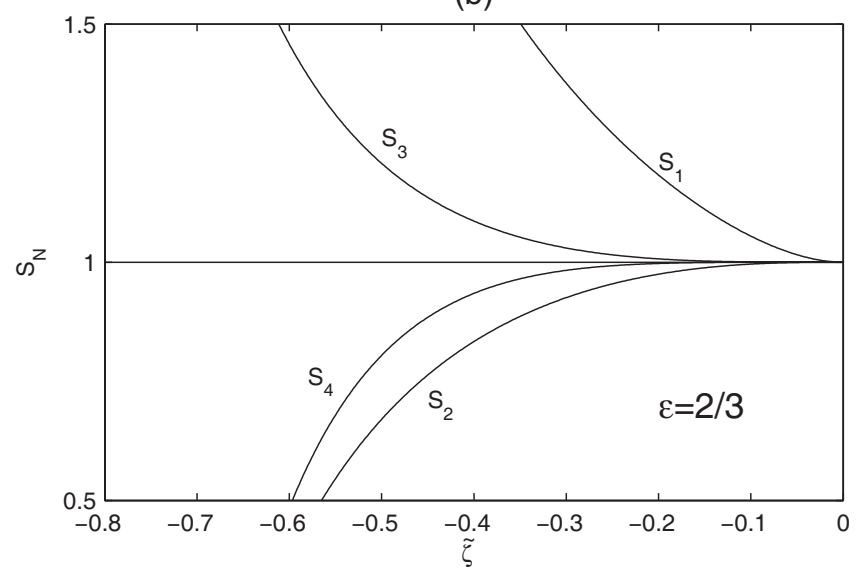

Fig. 2. Comparison of various truncations of the weakly-nonlinear approximation for the long wave phase speed with its exact expression $\tilde{c}_{E}$. (a) $\epsilon=1 / 3$; (b) $\epsilon=2 / 3$.

to reliably describe wave motions over a wide range of amplitudes.

\subsection{Higher-order approximations}

Toward the objective of generating a polynomial approximation for $\tilde{c}_{E}(\zeta ; \epsilon)$, the truncated version of the weaklynonlinear expansion of $\tilde{c}_{E}$ is first compared to the exact expression in terms of the following quantity:

$S_{N}(\tilde{\zeta} ; \epsilon)=\frac{1-\sum_{k=1}^{N} \tilde{\alpha}_{k}(\epsilon) \tilde{\zeta}^{k}}{\tilde{c}_{E}(\tilde{\zeta} ; \epsilon)}$.

The deviation of $S_{N}(\tilde{\zeta} ; \epsilon)$ from unity measures the error contained in the different truncations of the power series expansion, and provides a basis for defining the range of validity of the widely-used KdV1 and KdV2 versions defined by Eq. (1). Values of the function $S_{N}(\tilde{\zeta} ; \epsilon)$ for several values of $\epsilon$ are shown in Fig. 2 for different choices of the truncation parameter $N$. It is clear that KdV2 (i.e., the $S_{2}$ curve in Fig. 2) extends considerably the range of wave amplitudes over KdV1 (i.e., the $S_{1}$ curve) where quantitative descriptions are sought. Nevertheless, the approximate representation of the long-wave phase speed diverges quite rapidly from the true nonlinear value at wave amplitudes that may be well below those observed in a number of contexts. One alternative to extending the weakly-nonlinear expansion of $\tilde{c}_{E}$ to be used in the evolution equation, without resorting to an excessively high order truncation of the series representation for $\tilde{c}_{E}(\tilde{\zeta} ; \epsilon)$, is to seek some approximate summation of the series. To this end, the power-series expansion of the expression in Eq. (6) has been developed to high order and arranged in the following form:

$$
\begin{aligned}
\tilde{c}_{E}= & 1-\tilde{\alpha}_{1} \tilde{\zeta}-\tilde{\alpha}_{2} \tilde{\zeta}^{2} \\
& -\tilde{\alpha}_{3} \tilde{\zeta}^{3}\left\{1+\frac{5}{4} \tilde{\psi}+\frac{3}{2} \tilde{\psi}^{2}+\frac{7}{4} \tilde{\psi}^{3}+2 \tilde{\psi}^{4}+\cdots\right. \\
& +\frac{1}{4} \tilde{\phi}\left[1+\frac{7}{2} \tilde{\psi}+8 \tilde{\psi}^{2}+16 \tilde{\psi}^{3}+\cdots\right] \\
& \left.+\frac{1}{8} \tilde{\phi}^{2}\left[1+\frac{45}{8} \tilde{\psi}+\cdots\right]+\cdots\right\} .
\end{aligned}
$$

The quantities $(\tilde{\psi}, \tilde{\phi})$ appearing in this expression are defined as

$$
\tilde{\psi}=\frac{\epsilon \tilde{\zeta}}{1-\epsilon^{2}} ; \quad \tilde{\phi}=\frac{\tilde{\zeta}^{2}}{\left(1-\epsilon^{2}\right)^{2}} .
$$

Examination of this series suggests that any practical truncation of $\tilde{c}_{E}$ in polynonnmial form must include a term of order $\tilde{\zeta}^{3}$. With this in view, and motivated by the desire to produce an evolutionary model with polynomial nonlinearity, we propose using the "summed-series" $\tilde{c}_{S}$ for the nonlinear phase speed

$\tilde{c}_{S}=1-\tilde{\alpha}_{1} \tilde{\zeta}-\tilde{\alpha}_{2} \tilde{\zeta}^{2}-\tilde{\alpha}_{3 S} \tilde{\zeta}^{3}$,

yielding the extended evolution equation, termed here as KdV3S, with a quartic nonlinearity.

The coefficient $\tilde{\alpha}_{3 S}$ of the quartic nonlinear term in Eq. (14) is a "modeled" version of the coefficient $\tilde{\alpha_{3}}$, a modification to $\tilde{\alpha_{3}}$ that is designed to capture in approximate form the higher-order terms in Eq. (14) for $\tilde{c}_{E}(\tilde{\zeta} ; \epsilon)$. The modeled expression for $\tilde{\alpha}_{3 S}$ is

$$
\begin{aligned}
& \tilde{\alpha}_{3 S}=\frac{3}{2} \frac{\epsilon}{\left(1-\epsilon^{2}\right)^{3}} \exp \left\{-K_{S} \frac{\left|\epsilon \tilde{\zeta}_{S}\right|}{1-\epsilon^{2}}\right\} \times \\
& \exp \left\{\frac{\tilde{\zeta}_{S}^{2}}{\left(1-\epsilon^{2}\right)^{2}}\right\},
\end{aligned}
$$

which is obtained via a "pivoting" of the summed portion of the infinite series about a selected value of the wave amplitude, noted here as $\tilde{\zeta}_{S}$. The different series in Eq. (12) have a structure reminiscent of the exponential function, albeit with coefficients that clearly do not decay as $1 / n$ !. A factor $K_{S}$ is introduced to capture a single, "respectable summation" 
for the different series involving $\tilde{\psi}$ terms. After some testing of different parametric choices, a recommended set of values for the two parameters are $\tilde{\zeta}_{S}=1 / 3$ and $K_{S}=7 / 3$. Clearly these choices are somewhat arbitrary, and alternate values may yield a better fit for any given value of $\epsilon$, yet we suggest that the selected values yield a "respectable fit" encompassing a significant and physically-relevant range of depth ratios. The modeled form $\tilde{c}_{S}(\tilde{\zeta} ; \epsilon)$ of the phase speed for two depth ratios over a range of wave amplitudes is shown in Fig. 3 by the curve noted as KdV3S. Results in Fig. 3 are presented as a ratio of a particular approximation for the nonlinear phase speed divided by the exact value $\tilde{c}_{E}$. Hence, departures from unity provide an immediate measure the relative merits of any modeled approximation, and the range of amplitudes where its representation of the true nonlinear steepening is reasonably captured. The modeled expression $\tilde{c}_{S}$, with its selected fit parameters, significantly extends the range of amplitudes where a polynomial expression provides a good representation for the nonlinear steepening effects over that of KdV2, for example, but the fidelity of the fit degrades as the value of $\epsilon$ increases toward unity.

\subsection{A full-range, quadratic approximation - KdV2N}

Another approach toward developing an evolution model capable of describing strongly nonlinear long waves with reasonable fidelity, yet possessing polynomial nonlinearity is to construct a quadratic fit to the fully-nonlinear relation for $\tilde{c}_{E}(\tilde{\zeta}, \epsilon)$. To this end certain characteristic points on the curve representing the true function can be identified, and then a limited polynomial is proposed that passes through these points, thereby providing an approximate representation encompassing a wide range of wave amplitudes. The specific points vital to characterizing the function $\tilde{c}_{E}(\tilde{\zeta}, \epsilon)$ are the following: the intercept value $\left(\tilde{c}_{E}=1, \tilde{\zeta}=0\right)$, the maximum value $\left(\tilde{c}_{E}=\tilde{c}_{E m}, \tilde{\zeta}=\tilde{\zeta}_{m}\right)$, and the zero-crossing value $\left(\tilde{c}_{E}=0, \tilde{\zeta}_{00}\right)$. Using Eq. (6), the following quantities can be derived:

$$
\begin{aligned}
& \tilde{c}_{m}=1+\epsilon^{2} S_{c}(\epsilon), \\
& S_{c}(\epsilon)=\frac{3}{2}\left[1-\epsilon^{2}+\sqrt{1-\epsilon^{2}}\right]^{-1} ; \\
& \tilde{\zeta}_{m}=-\epsilon S_{m}(\epsilon), \\
& S_{m}(\epsilon)=1-\frac{1}{\sqrt{2}}\left[1+\sqrt{1-\epsilon^{2}}\right]^{-\frac{1}{2}} ; \\
& \tilde{\zeta}_{00}=-\operatorname{sgn}(\epsilon) S_{00}(\epsilon), \\
& S_{00}(\epsilon)=|\epsilon|+\frac{1}{\sqrt{3}}\left[1+\frac{\epsilon^{2}}{2}-\frac{1}{2} \sqrt{8 \epsilon^{2}+\epsilon^{4}}\right]^{\frac{1}{2}} .
\end{aligned}
$$

A quadratic approximation can only enforce passage through two points on the true function. Since the approximation should apply for all values of $\epsilon$, including $\epsilon=0$, we choose for the two pairs of $\left(\tilde{c}_{E}, \tilde{\zeta}\right)$, the intercept value $(1,0)$ and the max- (a)

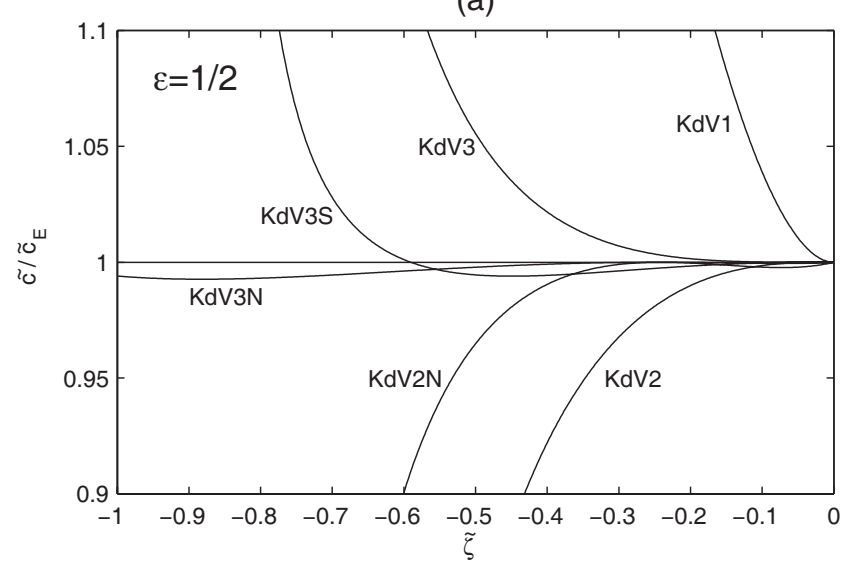

(b)

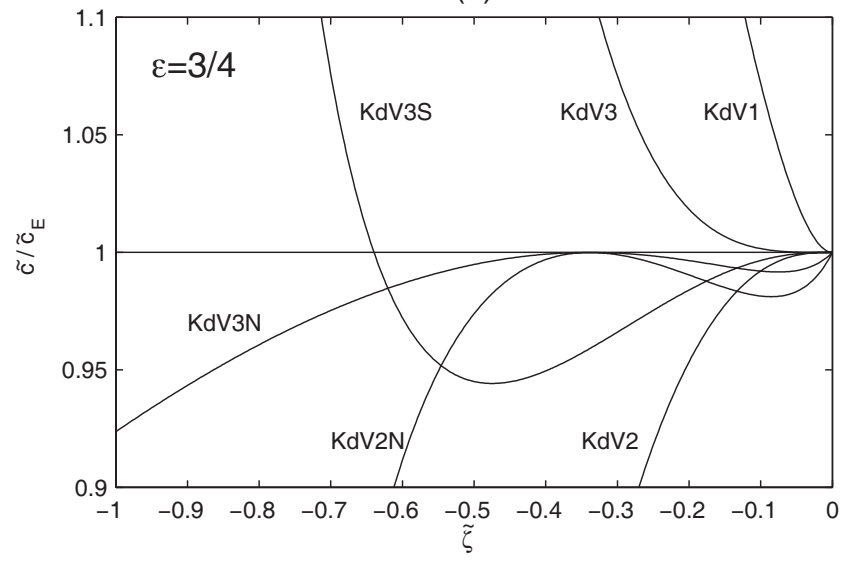

Fig. 3. Comparison of the various polynomial approximations for the phase speed underlying the different evolution models. (a) $\epsilon=1 / 2$; (b) $\epsilon=3 / 4$.

imum value $\left(\tilde{c}_{E m}, \tilde{\zeta}_{m}\right)$. This leads to the following quadratic fit, termed $\tilde{c}_{2 N}$,

$\tilde{c}_{2 N}=1-\tilde{\kappa}_{1} \tilde{\zeta}-\tilde{\kappa}_{2} \tilde{\zeta}^{2}$,

where the coefficients $\tilde{\kappa}_{1}$ and $\tilde{\kappa}_{2}$ have the values

$$
\begin{gathered}
\tilde{\kappa}_{1}=2 \epsilon \frac{S_{c}(\epsilon)}{S_{m}(\epsilon)}, \\
\tilde{\kappa}_{2}=\frac{S_{c}(\epsilon)}{S_{m}^{2}(\epsilon)} .
\end{gathered}
$$

This approximate representation for the nonlinear, long-wave phase speed is plotted on the right-hand portion of Fig. 1, shown by the dashed lines, to exhibit its correspondence with the true function. By construction, it matches the curve for $\epsilon=0$ exactly, and its deviation from the true function increases for increasing values of $\epsilon$ (i.e., disparate layer depths) and at higher amplitudes. The error is depicted for two values of $\epsilon$ by the curve noted KdV2N in Fig. 3. This quadratic fit clearly increases the range of wave amplitudes beyond $\mathrm{KdV} 2$, the weakly nonlinear quadratic approximation, but it 


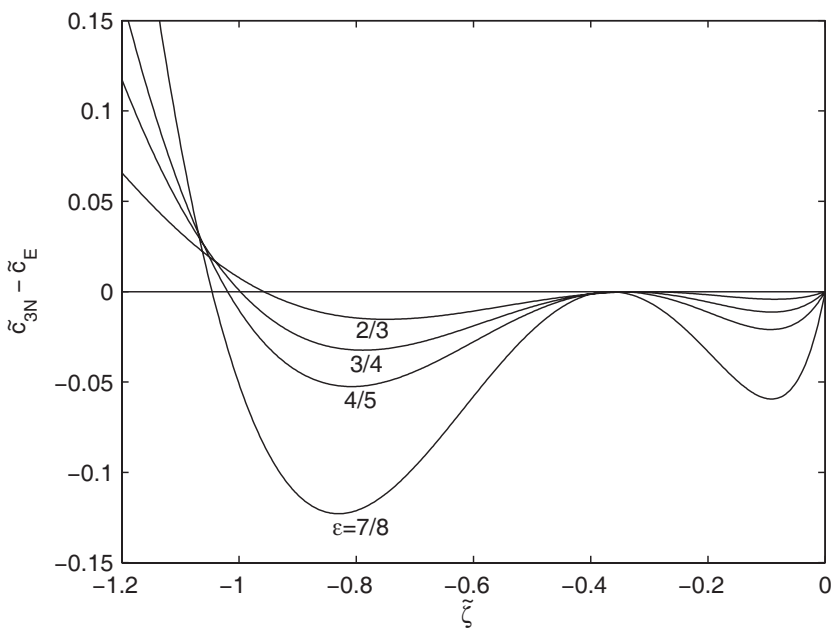

Fig. 4. The phase speed error for the $\mathrm{KdV} 3 \mathrm{~N}$ model relative to $\tilde{c}_{E}$ for different depth ratios.

does not provide advantages over KdV3S except that it is a lower order polynomial approximation.

\subsection{A full-range, cubic approximation - KdV3N}

Examination of the quadratic fit exhibited on the right-hand side of Fig. 1 shows that the fidelity of the quadratic representation for $\tilde{c}_{E}(\tilde{\zeta} ; \epsilon)$ degrades significantly at large amplitudes and higher values of $\epsilon$, and that a reasonably faithful approximation requires at least a polynomial of cubic order. To this point, we propose a cubic approximation that, in addition to passing through the points included in defining the quadratic fit (Eq. 19), includes enforcement of the zero-crossing value $\left(\tilde{c}_{E}=0, \tilde{\zeta}_{00}\right)$. This leads to the approximate relation, denoted by $c_{3 N}$,

$\tilde{c}_{3 N}=1-\tilde{\gamma}_{1} \tilde{\zeta}-\tilde{\gamma}_{2} \tilde{\zeta}^{2}-\tilde{\gamma}_{3} \tilde{\zeta}^{3}$,

where the coefficients $\tilde{\gamma}_{i}$ are given by the following functions of $\epsilon$ :

$$
\begin{aligned}
\tilde{\gamma}_{1}=\frac{\epsilon}{S_{00}}\left\{\frac{S_{c}}{S_{m}}\left(2 S_{00}+|\epsilon| S_{m}\right)\right. & \\
& \left.-|\epsilon| \frac{S_{m}^{2}\left(1+\epsilon^{2} S_{c}\right)}{\left(S_{00}-|\epsilon| S_{m}\right)^{2}}\right\},
\end{aligned}
$$

$\tilde{\gamma}_{2}=\frac{1}{S_{00}}\left\{\frac{S_{c}}{S_{m}^{2}}\left(S_{00}+2|\epsilon| S_{m}\right)\right.$

$$
\left.-2|\epsilon| \frac{S_{m}\left(1+\epsilon^{2} S_{c}\right)}{\left(S_{00}-|\epsilon| S_{m}\right)^{2}}\right\},
$$$$
\tilde{\gamma}_{3}=\operatorname{sgn}(\epsilon) \frac{S_{c}}{S_{00} S_{m}^{2}}\left\{1-\frac{S_{m}^{2}\left(1+\epsilon^{2} S_{c}\right)}{S_{c}\left(S_{00}-|\epsilon| S_{m}\right)^{2}}\right\} \text {. }
$$

This cubic fit is shown by the dash-dot curves on the lefthand side of Fig. 1. The match of this cubic fit with the true function appears remarkably good. The ratio of this approximation to the true function is depicted by the curves denoted $\mathrm{KdV} 3 \mathrm{~N}$ in Fig. 3. The reader will note that the ratio $\tilde{c}_{3 N} / \tilde{c}_{E}$ does not pass through the zero-crossing point $\tilde{\zeta}_{00}$. This occurs because the cubic approximation cannot enforce a simultaneous matching of the value of the functions and their slopes at the zero-crossing point, and consequently the ratio is necessarily finite at $\tilde{\zeta}_{00}$. However, in order to portray the high fidelity of the cubic approximation over the whole range of relevant wave amplitudes we present the difference between these functions $\Delta \tilde{c}_{3 N E}$ (defined as the difference $\left.\tilde{c}_{3 N}-\tilde{c}_{E}\right)$ in Fig. 4 for a wide range of depth ratios.

3.4 A full-range, cubic approximation with nonlinear dispersion $-\mathrm{KdV} 3 \mathrm{~N} \beta$

Thus far the proposed models for strongly nonlinear evolution have neglected nonlinear dispersive effects. Following Ostrovsky and Grue (2003), nonlinear dispersion can be incorporated, at least in a modeling sense, by replacing the fixed ambient depths of the two layers with their local values in the linear expression for the first dispersive correction, and by replacing the constant value of the linear, long-wave phase speed $c_{0}$ by its nonlinear, amplitude-dependent expression $\tilde{c}_{E}(\tilde{\zeta})$. That is, the fully-nonlinear evolution of long waves along a single characteristic can be written as

$\zeta_{t}+c_{0} \tilde{c}_{E}(\tilde{\zeta}) \zeta_{x}+\beta_{0} c_{0}\left(\tilde{\beta}(\tilde{\zeta}) \tilde{c}_{E}(\tilde{\zeta}) \zeta_{x x}\right)_{x}=0$,

with $\tilde{c}_{E}$ given by Eq. (6) and $\tilde{\beta}$ given by

$\tilde{\beta}(\tilde{\zeta})=\frac{\left(h_{1}-\zeta\right)\left(h_{2}+\zeta\right)}{h_{1} h_{2}}=\frac{1-(\epsilon+\tilde{\zeta})^{2}}{1-\epsilon^{2}}$.

The functional dependence of the product $\tilde{D}_{E}=\tilde{\beta} \tilde{c}_{E}$ on the local displacement $\tilde{\zeta}$ is somewhat complicated, having the form shown in Fig. 5. As argued further in the next section, it is of some interest to represent $\tilde{D}_{E}$ by an approximate quadratic dependence on $\tilde{\zeta}$. This objective is readily accomplished by constructing a quadratic form that passes through the intercept value $\left(\tilde{D}_{E}=0, \tilde{\zeta}=0\right)$ and the maximum value $\tilde{D}_{E}\left(\tilde{\zeta}_{D_{m}}\right)=\tilde{D}_{E m}$ of the function $\tilde{D}_{E}$. To that end we propose the approximate form for the nonlinear dispersive term

$$
\begin{aligned}
\tilde{D}_{E}(\tilde{\zeta}) & =\tilde{\beta}(\tilde{\zeta}) \tilde{c}_{E}(\tilde{\zeta}) \\
& \simeq \tilde{D}(\tilde{\zeta})=D_{m}\left\{1-\frac{\left(\tilde{\zeta}-\tilde{\zeta}_{D m}\right)^{2}}{d_{m}^{2}}\right\} .
\end{aligned}
$$

One can readily verify that excellent approximations for the "fit" parameters $\left(D_{m}, \tilde{\zeta}_{D_{m}}, d_{m}\right)$ are:

$D_{m}=\frac{1+\epsilon^{2}}{1-\epsilon^{2}}, \quad \tilde{\zeta}_{D_{m}}=-\frac{3}{5} \epsilon, \quad d_{m}=\frac{3 \sqrt{2}}{10} \sqrt{1+\epsilon^{2}}$.

This quadratic approximation $\tilde{D}$ is compared with the exact relation $\tilde{D}_{E}$ on the left half of Fig. 5 . 
The evolution equation employing Eq. (22), and when incorporating the quadratic approximation for the proposed nonlinear dispersive term Eq. (28) in accordance with the extended form put forward in Eq. (26), is referred to here as $\operatorname{KdV} 3 \mathrm{~N} \beta_{c}$. We note, however, and as is readily evident from Fig. 5, both the function $\tilde{D}_{E}$ or its approximation $\tilde{D}$ become negative at large amplitudes. The sign of the dispersive term, therefore, may pass through zero and change sign as the local displacement becomes large. As an attempt to avoid this deficiency in the $\mathrm{KdV} 3 \mathrm{~N} \beta_{c}$ model, we propose an alternate approximate form for the nonlinear dispersive effect. This alternate form simply employs a constant value $\tilde{c}_{E}=1$ in Eq. (28), since it is the behavior of $\tilde{c}_{E}$ that drives the value of $\tilde{D}_{E}$ toward zero as the amplitude increases. Hence, the functional form for $\tilde{D}$ is given simply by the expression in Eq. (27), a form which is exhibited on the right half of Fig. 5. This approximation for the nonlinear dispersion effect yields an evolution model denoted here as $\operatorname{KdV} 3 \mathrm{~N} \beta_{1}$.

\section{Comparison of evolution models}

A hierarchy of evolutionary models belonging generally to the KdV family has been set forward in the preceding section, each with a differing potential for describing stronglynonlinear wave motions. In this section we seek to provide bases for discriminating between possible advantages or limitations of the various models by computing several solution properties of these models.

The first solution property to be compared is that of isolated solitary wave solutions admitted by the equations. For purposes of this discussion, we represent the extended version of $\mathrm{KdV}$ equation in the general form as given in Eq. (26). The proposed, nonlinear characterization of the dispersive term as given in Eq. (26) is included and, as noted earlier, the dimensionless amplitude is $\tilde{\zeta}=\zeta / h$. A solitary wave is a traveling wave solution given functionally by an expression of the form

$\tilde{\zeta}(\tilde{x}, \tilde{t})=\tilde{Z}(\tilde{X})=\tilde{Z}\left(\sqrt{\frac{h^{2}}{\beta_{0}}}(\tilde{x}-\tilde{V} \tilde{t})\right)$.

In this expression we employ the definitions $(x, t)=\left(h \tilde{x}, h \tilde{t} / c_{0}\right)$, and $\tilde{Z}(\tilde{X})$ satisfies the nonlinear eigenvalue problem

$\frac{d^{2} \tilde{Z}}{d \tilde{X}^{2}}=\frac{1}{\tilde{D}_{E}(\tilde{Z})}\{\tilde{v} \tilde{Z}-\tilde{U}(\tilde{Z})\} \equiv R(\tilde{Z})$.

The eigenvalue $\tilde{v}=\tilde{V}-1$ (where the dimensional solitary wave speed is given by $\left.V=c_{0} \tilde{V}\right)$ is that function of the wave amplitude such that $\tilde{Z}(\tilde{X})$ satisfies homogeneous boundary conditions $\tilde{Z}(+\infty)=\tilde{Z}(-\infty)=0$. The function $\tilde{D}_{E}(\tilde{Z})$ is given in Eq. (28), while $\tilde{U}(\tilde{Z})$ is given by the relation

$\tilde{U}(\tilde{Z})=\int_{0}^{\tilde{Z}}\left\{\tilde{c}_{E}(\tilde{\zeta})-1\right\} d \tilde{\zeta}$.

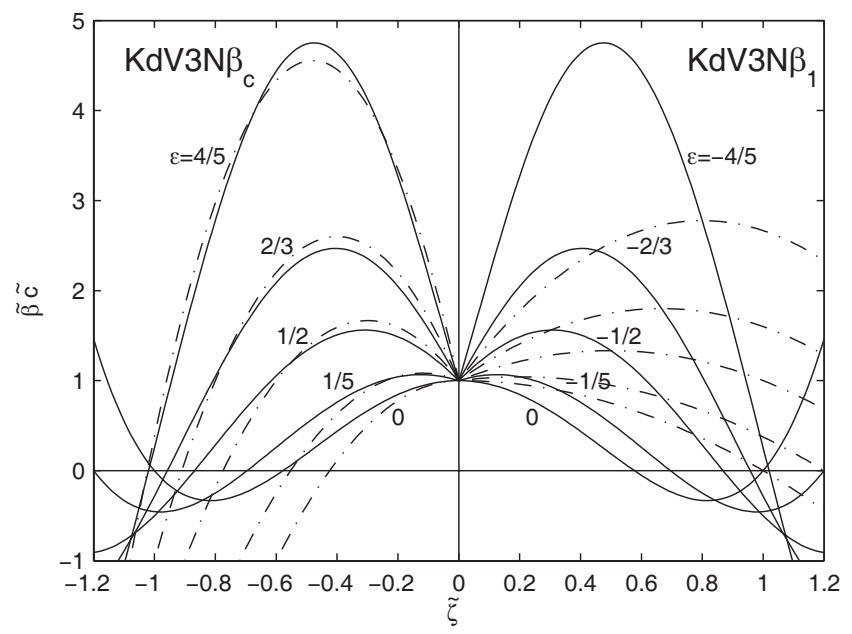

Fig. 5. Comparison of different models for nonlinear effects of dispersion.

The eigenvalue corresponding to solitary wave solutions can be obtained directly by multiplying Eq. (32) by $d \tilde{Z} / d \tilde{X}$ and writing the equation as

$\frac{d}{d \tilde{X}}\left\{\frac{1}{2}\left(\frac{d \tilde{Z}}{d \tilde{X}}\right)^{2}-\int^{\tilde{Z}} R(\theta) d \theta\right\}=0$.

Since $d \tilde{Z} / d \tilde{X}=0$ at the extremum of the solitary wave (i.e., crest or trough), one obtains directly the following expression for the eigenvalue $\tilde{v}$ in terms of the solitary wave amplitude $\tilde{A}$ :

$\tilde{v}=\frac{\int_{0}^{\tilde{A}} \tilde{U}(\tilde{Z}) / \tilde{D}_{E}(\tilde{Z}) d \tilde{Z}}{\int_{0}^{\tilde{A}} \tilde{Z} / \tilde{D}_{E}(\tilde{Z}) d \tilde{Z}}$.

We consider several cases of the eigenvalue relation given in Eq. (34). First, we consider the case, termed $\operatorname{KdVE} \beta_{c}$, with the exact nonlinear phase speed and the exact expression for $\tilde{D}_{E}(\tilde{Z})$. The variation of the eigenvalue (alt., the solitary wave speed) with wave amplitude, as computed from Eq. (34), is presented in Fig. 6 for the particular depth ratio corresponding to $\epsilon=2 / 3$. This exact result is compared in Fig. 6 with various alternate evolution models defined in the previous section. Analytic expressions for the eigenvalue for the different models are summarized in Appendix A. Fig. 6 shows that the eigenvalue for KdV1 provides a reasonable approximation for the speed of nonlinear wave features only for wave amplitudes $|\tilde{\zeta}|<0.1$. Further, KdV2 provides a useful approximation for wave amplitudes satisfying roughly $|\tilde{\zeta}|<0.4$ (at this value of $\epsilon$. For larger amplitude waves, one of the higher-order approximations discussed here is needed to give a faithful prediction of the wave speed. The reader will also note that, with the exception of KdV1, all models 


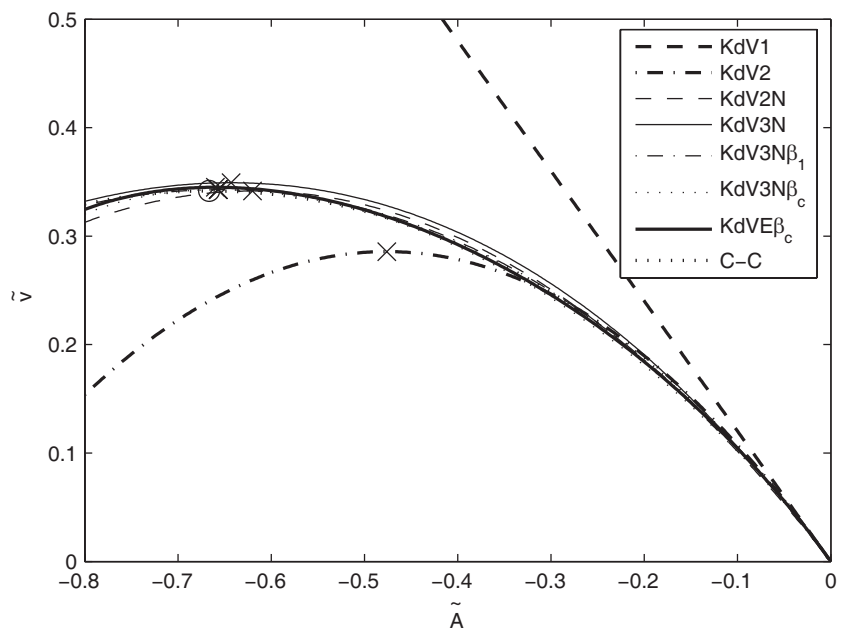

Fig. 6. Comparison of the dependence of the eigenvalue $\tilde{v}$ on solitary wave amplitude for $\epsilon=2 / 3$.

yield a maximum value for the eigenvalue (alt., solitary wave speed) at some intermediate amplitude. This point will be addressed further in a subsequent paragraph.

The next solution component of the various proposed evolution models that we compare is the profile of solitary waves. Profiles for specified amplitudes obtained using different evolution models are shown in Fig. 7 for the particular depth ratio given by $\epsilon=2 / 3$. As expected, profile forms differ only marginally at low amplitudes, but significant disparities emerge as the amplitude increases. At low amplitudes the wave width diminishes with increasing amplitude for all models, a result that holds uniformly for KdV1, as is well known. However, as the amplitude increases (alt., as the degree of nonlinearity becomes increasingly significant), all models with the sole exception of KdV1 manifest a reversal in this trend of wave width increasing with wave amplitude. In particular, the profile obtained using KdV2 becomes quite wide with a flat trough when the amplitude is $\tilde{A}=-0.47$. This amplitude is just marginally below the limiting amplitude $\tilde{A}_{\text {lim }}=-\tilde{\mu}_{1} / \tilde{\mu}_{2}=-0.47619 \cdots$ for KdV2. An analytical expression for this limiting amplitude is obtained in straightforward fashion from the functional relation for a solitary wave solution of KdV2

$$
\begin{aligned}
& \tilde{Z}(\tilde{X})=\tilde{A} \frac{1-\mathcal{T}^{2}}{1-b \mathcal{T}^{2}} ; \quad \mathcal{T} \equiv \tanh (K \tilde{X}) ; \\
& b=-\frac{\tilde{\alpha}_{2} \tilde{A}}{2 \tilde{\alpha}_{1}+\tilde{\alpha}_{2} \tilde{A}} .
\end{aligned}
$$

The limiting wave amplitude corresponds to that for which the parameter $b$ approaches the value of unity from below.

As evident in Fig. 7, wave profiles for KdV2N and KdV3N at an amplitude of $\tilde{A}=-0.6$ are nearly coincident, but the profile of the wave predicted by $\mathrm{KdV} 1$ is significantly more narrow. This characteristic under-prediction of the width of the profile by $\mathrm{KdV} 1$ for higher wave amplitudes is wellknown based on established results using KdV2 models (e.g., Evans and Ford, 1996; Lamb and Wan, 1998; Grimshaw, 2002; Holloway and Pelinovsky, 2002). However, the results presented here suggest that the widening of the wave profile is significantly over-estimated by $\mathrm{KdV} 2$. The widening effect beyond that corresponding to KdV1 becomes significant only at amplitudes in excess of the limiting value for KdV2. We point out for emphasis that the amplitude $\tilde{\zeta}_{1}$ (i.e., the dimensionless wave amplitude based on the controlling dimension) for the particular case of $\epsilon=2 / 3$ corresponding to the computed profiles shown in Fig. $7 \mathrm{a}$ is $\tilde{\zeta}_{1}=-1.5$ when $\tilde{\zeta}=-0.5$, and that the wave profile predicted by $\mathrm{KdV} 1$ is actually a "not-so-unreasonable" approximation when compared with the profile based on KdV3N at this large amplitude state. The profile shown in the last panel of Fig. 7a for an amplitude of $\tilde{A}=-0.64$ does not include data for KdV2N because the amplitude exceeds the limiting value for this model. The widening effect for $\mathrm{KdV} 3 \mathrm{~N}$ relative to $\mathrm{KdV} 1$ at this amplitude is partially due to the fact that the amplitude $\tilde{A}=-0.64$ is only slightly below the value of $\tilde{A}_{\lim }$ for $\mathrm{KdV} 3 \mathrm{~N}$ at $\epsilon=2 / 3$.

As discussed above, the models excluding KdV1 possess limiting amplitudes which, when approached from below, define conditions where the wave profile broadens significantly, even to the point where the profile approaches a front separating two uniform states. This naturally introduces the notion of conjugate states as introduced by Benjamin (1966), and computed by Turner and Vanden-Broeck (1988) and described in further detail by Amick and Turner (1986) and by Evans and Ford (1996). Values of the limiting solitary wave amplitudes for the models $\mathrm{KdV} 2, \mathrm{KdV} 2 \mathrm{~N}, \mathrm{KdV} 3 \mathrm{~N}$, $\operatorname{KdV} 3 N \beta_{1}, \operatorname{KdV} 3 N \beta_{c}$ and $\operatorname{KdVE} \beta_{c}$ are presented in Table 2 for a range of depth ratios, and these limiting amplitudes are compared with results of the conjugate state amplitude computed using the theory of Amick and Turner (1986). For the special case of a two-layer stratification with a rigid upper surface, the environmental case under examination here, Amick and Turner (1986) obtained an analytic solution for the conjugate state. When the Boussinesq approximation is invoked, and when their results are transposed in terms of the non-dimensional variables used herein, the conjugate state is defined simply by the relations

$\tilde{\zeta}_{c s}=-\epsilon ; \quad \tilde{c}_{c s}^{2}=\frac{1}{1-\epsilon^{2}} ; \quad \tilde{u}_{c s 1}^{2}=\frac{1}{\tilde{u}_{c s 2}^{2}}=\frac{1-\epsilon}{1+\epsilon}$.

The quantities $\tilde{u}_{c s 1}$ and $\tilde{u}_{c s 2}$, also listed in Table 2, define the velocities of the upper and lower layers behind the front, as measured in a wave-fixed frame, respectively. These values provide a basis for evaluating the maximum shear (i.e., vortex sheet strength) at the interface when a wave reaches its limiting amplitude. The value of this velocity difference is important relative to estimating the potential for KelvinHelmholtz instability and internal mixing to occur as the wave passes. 
(a)
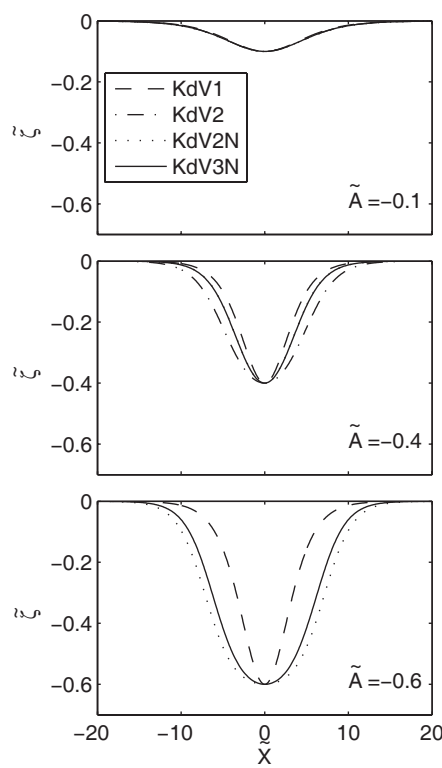
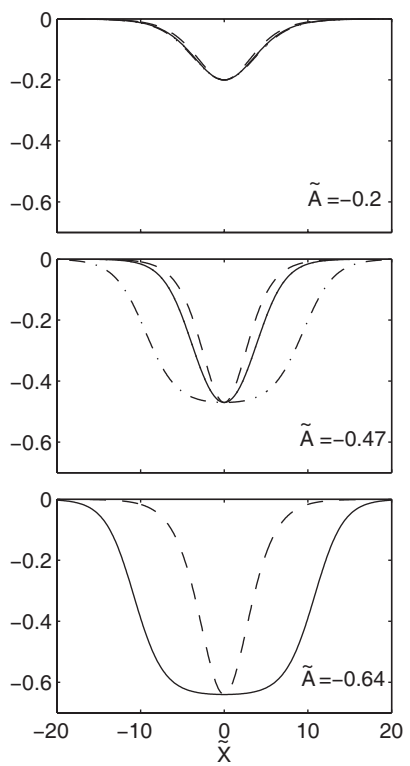

(b)
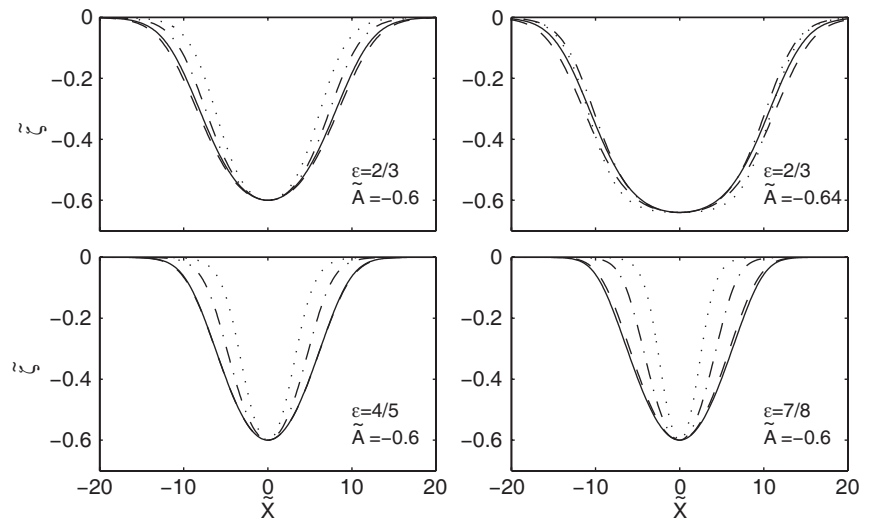

\begin{tabular}{|cc|}
$\cdots \cdots$ & $\operatorname{KdV} 3 N$ \\
- & $-\operatorname{KdV} 3 N \beta_{1}$ \\
- & $\operatorname{KdV} 3 N \beta_{c}$ \\
- & $\operatorname{KdVE} \beta_{\mathrm{c}}$ \\
\hline
\end{tabular}

Fig. 7. Comparison of solitary wave profile described by various evolution models at different amplitudes: (a) wave forms for $\epsilon=2 / 3$ (left six panels); (b) wave forms for different values of $\epsilon$ (right four panels).

Included in Table 2 is a comparison of the speed $\tilde{c}_{c S}$ of the "front" separating the conjugate states and the value of the speed $\tilde{c}_{E}\left(\tilde{\zeta}_{c s}\right)$ of the phase speed (Eq. 6) evaluated at the amplitude of the conjugate state. These values are particularly disparate for low values of the depth ratio parameter $\epsilon$, a difference that deserves some explanation. The computation of the conjugate state is based on an integral theory that requires only an evaluation of the pressure at the upstream and downstream locations relative to the front. As such, it makes no approximation regarding the pressure distribution through the front, and accurately uses the appropriate hydrostatic pressure distribution asymptotically far away from the front. On the other hand, the derivation of the phase speed given in Eq. (6) has explicitly used the leading order approximation for the pressure distribution in the limit of infinitely long waves everywhere through the wave profile, namely a hydrostatic distribution. This distinction naturally gives disparate values of the wave speed, and this different representation of the pressure is especially apparent in the case of more nearly equal layer depths. When the layer depths are widely disparate, the front dynamics implicit in the conjugate state solution are more nearly hydrostatic.

We return now to the eigenvalue data exhibited in Fig. 6, and inject an observation regarding the existence of limiting amplitudes of stationary solutions (e.g., solitary waves) of the various evolution models. It was noted following Eq. (35) that the amplitude corresponding to the maximum of the eigenvalue for $\mathrm{KdV} 2$ and $\mathrm{KdV} 2 \mathrm{~N}$ coincided with the condition that the parameter $b=1$, the condition for Eq. (35)

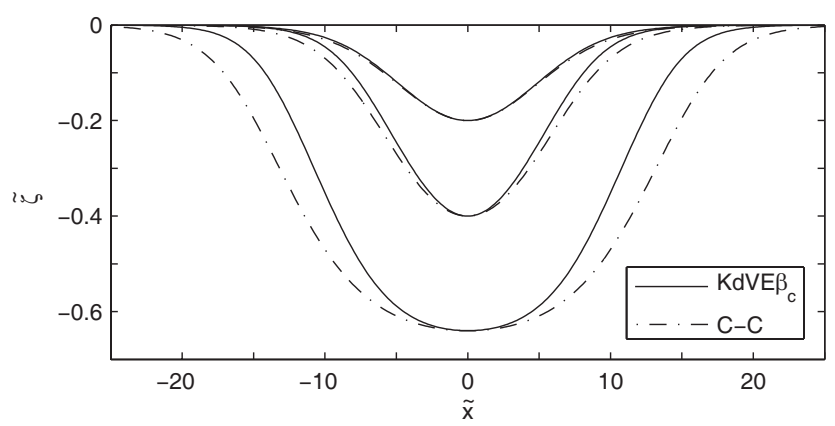

Fig. 8. Comparison of solitary wave profile described by $\operatorname{KdVE} \beta_{c}$ and Choi-Camassa's model for $\epsilon=2 / 3$.

to describe an isolated front. In fact, our computations reveal that the limiting amplitude condition for all evolution models corresponds to that amplitude for which the eigenvalue reaches a maximum. Of course, the eigenvalue for KdV1 has no local maximum and, therefore, $\mathrm{KdV} 1$ does not possess a limiting amplitude. For this reason, a mark denoting the peak of the eigenvalue is placed on the curves in Fig. 6, points that correspond to the limiting amplitudes for each of the models represented in the figure.

It is important to note that an exact, stationary solution of the Euler equations for an isolated (i.e., solitary) wave at the interface between two layers of homogeneous fluid was constructed by Choi and Camassa (1999). For the purpose of comparing their exact solution with stationary solutions 
Table 2. Limiting wave amplitudes for different evolution models, and comparison with the theory of conjugate states.

\begin{tabular}{cccccccccccc}
\hline & $\mathrm{KdV} 2$ & $\mathrm{KdV} 2 \mathrm{~N}$ & $\mathrm{KdV} 3 \mathrm{~N}$ & $\mathrm{KdV} 3 \mathrm{~N} \beta_{1}$ & $\mathrm{KdV} 3 \mathrm{~N} \beta_{c}$ & $\mathrm{KdVE} \beta_{c}$ & \multicolumn{5}{c}{ conjugate state } \\
\cline { 9 - 12 }$\epsilon$ & $\tilde{\zeta}_{\max }$ & $\tilde{\zeta}_{\max }$ & $\tilde{\zeta}_{\max }$ & $\tilde{\zeta}_{\max }$ & $\tilde{\zeta}_{\max }$ & $\tilde{\zeta}_{\max }$ & $\tilde{\zeta}_{\mathrm{cs}}$ & $\left.\tilde{c}_{E}\right|_{\zeta_{c s}}$ & $\tilde{c}_{c s}$ & $\tilde{u}_{c s 1}$ & $\tilde{u}_{c s 2}$ \\
\hline 0 & 0.000 & 0.000 & 0.000 & 0.000 & 0.000 & 0.000 & 0.000 & 1.000 & 1.000 & 1.000 & 1.000 \\
$1 / 5$ & -0.196 & -0.199 & -0.200 & -0.200 & -0.200 & -0.200 & -0.200 & 1.000 & 1.021 & 0.817 & 1.225 \\
$1 / 3$ & -0.314 & -0.328 & -0.331 & -0.333 & -0.333 & -0.333 & -0.333 & 1.000 & 1.061 & 0.707 & 1.414 \\
$1 / 2$ & -0.429 & -0.482 & -0.492 & -0.497 & -0.497 & -0.498 & -0.500 & 1.000 & 1.155 & 0.577 & 1.732 \\
$2 / 3$ & -0.476 & -0.620 & -0.643 & -0.657 & -0.657 & -0.660 & -0.667 & 1.000 & 1.342 & 0.447 & 2.236 \\
$3 / 4$ & -0.457 & -0.677 & -0.712 & -0.733 & -0.732 & -0.739 & -0.750 & 1.000 & 1.512 & 0.378 & 2.646 \\
$4 / 5$ & -0.424 & -0.706 & -0.749 & -0.777 & -0.775 & -0.786 & -0.800 & 1.000 & 1.667 & 0.333 & 3.000 \\
$5 / 6$ & -0.390 & -0.721 & -0.771 & -0.805 & -0.802 & -0.816 & -0.833 & 1.000 & 1.809 & 0.302 & 3.317 \\
$6 / 7$ & -0.359 & -0.729 & -0.785 & -0.823 & -0.820 & -0.837 & -0.857 & 1.000 & 1.942 & 0.277 & 3.606 \\
$7 / 8$ & -0.332 & -0.734 & -0.794 & -0.837 & -0.833 & -0.853 & -0.875 & 1.000 & 2.066 & 0.258 & 3.873 \\
$8 / 9$ & -0.308 & -0.737 & -0.800 & -0.846 & -0.842 & -0.865 & -0.889 & 1.000 & 2.183 & 0.243 & 4.123 \\
$9 / 10$ & -0.287 & -0.738 & -0.804 & -0.854 & -0.849 & -0.874 & -0.900 & 1.000 & 2.294 & 0.299 & 4.359 \\
\hline
\end{tabular}

of the evolution models proposed here, the wave profiles for moderate amplitude waves of $\operatorname{KdVE} \beta_{c}$ model are compared with the corresponding Choi-Camassa (C-C) wave for $\epsilon=2 / 3$ in Fig. 8. It is seen that the $\operatorname{KdVE} \beta_{c}$ profile slightly underestimates the width of the wave profile, a consequence (we conjecture) of inadequacies of the approximate representation of nonlinear dispersive effects in Eqs.(26) and (27). In regard to the speed and amplitudes of the limiting wave, the Choi-Camassa predictions are necessarily identical with the conjugate state values given in Table 2.

To compare the solitary wave profiles predicted by the various evolution models at a further level of detail, we evaluate the spatial location $\tilde{X}_{i}$ of the inflection point of the profile, the wave amplitude $\tilde{\zeta}_{i}$ at the inflection point, and the wave slope $\tilde{\zeta}_{\tilde{X}_{i}}$ at the inflection point. These data are presented in Table 3 for profiles computed using the depth ratio corresponding to $\epsilon=2 / 3$.

Data do not appear in some columns because the selected amplitude in the leading column exceeds the limiting amplitude for the particular model. The wave width, as represented by the location of the inflection point $\tilde{X}_{i}$, diminishes with increasing wave amplitude in all models when the amplitude is small, but most emphatically for KdV1. In the models other than $\mathrm{KdV} 1$, the width begins to increase with increasing wave amplitude beyond modest amplitudes of the order $\tilde{\zeta}_{0} \approx-0.3$. The increase in wave width becomes quite rapid as these profiles approach their limiting amplitudes.

The last column of Table 3 contains corresponding data obtained from Choi and Camassa's exact, stationary solution. One observes that $\mathrm{KdV} 3 \mathrm{~N} \beta_{c}$ model under estimates the wave width by about $7 \%$ at an amplitude of $\tilde{\zeta}_{0}=0.4$, but the wave speeds are essentially identical. Hence, the $\operatorname{KdV} 3 \mathrm{~N} \beta_{c}$ model, for example, provides a nonlinear evolution equation for moderately large waves in which the position, phase relations and characteristics of individual wave profiles are represented with quite high fidelity. For point of reference, the value $\tilde{\zeta}_{0}=0.4$ corresponds to a wave amplitude $\tilde{\zeta}_{1}=-1.2$ for $\epsilon=2 / 3\left(h_{2}=5 h_{1}\right)$.

A third level of comparison of the different models is that of spatio-temporal evolution from a fixed initial condition. For this purpose, an initial condition corresponding to a "non-equilibrium" form of a KdV2 solitary wave was chosen. That is, the profile defined by Eq. (35) was used, and the parameter set $(\tilde{A}=-1 / 2, b=3 / 4, K=1 / 4)$ was purposefully selected in order to yield more than one leading solitary wave in the asymptotic state for KdV2 and the selected depth ratio $\epsilon=2 / 3$. The result of a sequence of simulations on the semi-infinite line are shown in Fig. 9 where evolutionary data are shown for a fixed time in a spatial coordinate frame that moves with the linear, long-wave phase speed $c_{0}$. A further comparison of the evolution leading to the wave forms shown in Fig. 9 is obtained by comparing the temporal variation of the amplitude of the leading wave in the packet, and its spatial position, as a function of time. These results are plotted in Fig. 10.

Another point of interest regarding strongly nonlinear evolution in realistic environments pertains to the propagation in a wave guide of variable depth. The effect of variable depth in the KdV1 or KdV2 models for the two-layer stratification involves the addition of the term $\frac{1}{2} \zeta d c_{0} / d x$ in Eq. (1). A model that incorporates the effect of strong nonlinearity can be postulated by extending the weakly-nonlinear model to contain the following generalization of Eq. (26): 
Table 3. Comparison of solitary wave inflection point data for different evolution models.

\begin{tabular}{l|ccc|ccc|ccc|ccc}
\hline & $\tilde{K}_{i}$ & $\tilde{K}_{i} 1$ & $\tilde{\zeta}_{\tilde{X}_{i}}$ & $\tilde{X}_{i}$ & $\tilde{\zeta}_{i}$ & $\tilde{\zeta}_{\tilde{X}_{i}}$ & $\tilde{X}_{i}$ & $\tilde{\zeta}_{i}$ & $\tilde{\zeta}_{\tilde{X}_{i}}$ & $\tilde{X}_{i}$ & $\tilde{\zeta}_{i}$ & $\tilde{\zeta}_{\tilde{X}_{i}}$ \\
\hline-0.1 & 3.80 & -0.067 & 0.013 & 4.32 & -0.066 & 0.012 & 4.36 & -0.066 & 0.012 & 4.31 & -0.066 & 0.012 \\
-0.15 & 3.10 & -0.100 & 0.024 & 3.81 & -0.098 & 0.021 & 3.77 & -0.098 & 0.021 & 3.74 & -0.098 & 0.021 \\
-0.2 & 2.69 & -0.133 & 0.038 & 3.62 & -0.128 & 0.030 & 3.47 & -0.130 & 0.031 & 3.46 & -0.130 & 0.031 \\
-0.25 & 2.40 & -0.167 & 0.053 & 3.60 & -0.158 & 0.040 & 3.32 & -0.161 & 0.041 & 3.34 & -0.160 & 0.041 \\
-0.3 & 2.20 & -0.200 & 0.069 & 3.76 & -0.185 & 0.048 & 3.28 & -0.191 & 0.051 & 3.31 & -0.190 & 0.051 \\
-0.35 & 2.03 & -0.233 & 0.087 & 4.12 & -0.208 & 0.055 & 3.33 & -0.219 & 0.061 & 3.36 & -0.218 & 0.061 \\
-0.4 & 1.90 & -0.267 & 0.107 & 4.87 & -0.226 & 0.060 & 3.47 & -0.245 & 0.070 & 3.51 & -0.245 & 0.070 \\
-0.45 & 1.79 & -0.300 & 0.127 & 6.74 & -0.237 & 0.063 & 3.73 & -0.269 & 0.079 & 3.75 & -0.268 & 0.077 \\
-0.47 & 1.75 & -0.313 & 0.136 & 9.42 & -0.238 & 0.064 & 3.88 & -0.277 & 0.081 & 3.89 & -0.277 & 0.080 \\
-0.5 & 1.70 & -0.333 & 0.149 & - & - & - & 4.18 & -0.288 & 0.084 & 4.16 & -0.288 & 0.084 \\
-0.55 & 1.62 & -0.367 & 0.172 & - & - & - & 4.99 & -0.302 & 0.088 & 4.83 & -0.304 & 0.088 \\
-0.6 & 1.55 & -0.400 & 0.196 & - & - & - & 7.09 & -0.309 & 0.090 & 6.16 & -0.313 & 0.091 \\
-0.64 & 1.50 & -0.427 & 0.216 & - & - & - & - & - & - & 10.8 & -0.316 & 0.092 \\
-0.65 & 1.49 & -0.433 & 0.221 & - & - & - & - & - & - & - & - & - \\
\hline
\end{tabular}

Table 3. Continued.

\begin{tabular}{|c|c|c|c|c|c|c|c|c|c|c|c|c|}
\hline \multirow[b]{2}{*}{$\tilde{\zeta}_{0}$} & \multicolumn{3}{|c|}{$\mathrm{KdV} 3 \mathrm{~N} \beta_{1}$} & \multicolumn{3}{|c|}{$\operatorname{KdV} 3 \mathrm{~N} \beta_{c}$} & \multicolumn{3}{|c|}{$\operatorname{KdVE} \beta_{c}$} & \multicolumn{3}{|c|}{$\mathrm{C}-\mathrm{C}$} \\
\hline & $\tilde{X}_{i}$ & $\tilde{\zeta}_{i}$ & $\tilde{\zeta}_{\tilde{X}_{i}}$ & $\tilde{X}_{i}$ & $\tilde{\zeta}_{i}$ & $\tilde{\zeta}_{\tilde{X}_{i}}$ & $\tilde{X}_{i}$ & $\tilde{\zeta}_{i}$ & $\tilde{\zeta}_{\tilde{X}_{i}}$ & $\tilde{X}_{i}$ & $\tilde{\zeta}_{i}$ & $\tilde{\zeta}_{\tilde{X}_{i}}$ \\
\hline-0.1 & 4.74 & -0.065 & 0.011 & 5.56 & -0.063 & 0.010 & 5.33 & -0.063 & 0.011 & 5.20 & -0.064 & 0.010 \\
\hline-0.15 & 4.27 & -0.096 & 0.020 & 5.19 & -0.093 & 0.017 & 4.98 & -0.093 & 0.018 & 4.90 & -0.095 & 0.017 \\
\hline-0.2 & 4.07 & -0.126 & 0.028 & 5.05 & -0.122 & 0.024 & 4.88 & -0.122 & 0.025 & 4.86 & -0.124 & 0.024 \\
\hline-0.25 & 4.02 & -0.155 & 0.037 & 5.04 & -0.150 & 0.031 & 4.89 & -0.150 & 0.032 & 4.96 & -0.153 & 0.031 \\
\hline-0.3 & 4.07 & -0.183 & 0.045 & 5.11 & -0.177 & 0.038 & 4.98 & -0.176 & 0.039 & 5.17 & -0.179 & 0.037 \\
\hline-0.35 & 4.20 & -0.209 & 0.053 & 5.26 & -0.203 & 0.044 & 5.13 & -0.202 & 0.045 & 5.48 & -0.205 & 0.042 \\
\hline-0.4 & 4.42 & -0.234 & 0.060 & 5.48 & -0.228 & 0.050 & 5.36 & -0.227 & 0.051 & 5.89 & -0.228 & 0.047 \\
\hline-0.45 & 4.75 & -0.256 & 0.066 & 5.82 & -0.251 & 0.055 & 5.69 & -0.249 & 0.056 & 6.45 & -0.249 & 0.051 \\
\hline-0.47 & 4.93 & -0.264 & 0.068 & 5.99 & -0.259 & 0.057 & 5.86 & -0.258 & 0.058 & 6.73 & -0.256 & 0.052 \\
\hline-0.5 & 5.26 & -0.275 & 0.071 & 6.31 & -0.271 & 0.059 & 6.16 & -0.270 & 0.061 & 7.22 & -0.266 & 0.054 \\
\hline-0.55 & 6.04 & -0.290 & 0.075 & 7.09 & -0.287 & 0.062 & 6.89 & -0.286 & 0.064 & 8.34 & -0.280 & 0.057 \\
\hline-0.6 & 7.44 & -0.300 & 0.077 & 8.50 & -0.299 & 0.065 & 8.19 & -0.298 & 0.067 & 10.2 & -0.290 & 0.058 \\
\hline-0.64 & 10.3 & -0.303 & 0.078 & 11.4 & -0.303 & 0.065 & 10.7 & -0.303 & 0.067 & 13.3 & -0.2 & 0.059 \\
\hline-0.65 & 12.4 & -0.304 & 0.078 & 13.6 & -0.304 & 0.066 & 12.4 & -0.304 & 0.068 & 14.9 & -0.295 & 0.059 \\
\hline
\end{tabular}

$\zeta_{t}+\frac{1}{2} \frac{\partial c}{\partial x} \zeta+c_{0} \tilde{c}_{E}(\tilde{\zeta}) \zeta_{x}+\beta_{0} c_{0}\left(\tilde{\beta}(\tilde{\zeta}) \tilde{c}_{E}(\tilde{\zeta}) \zeta_{x x}\right)_{x}=0$

In this equation we intend that evaluation of the rarefaction term $\partial c / \partial x$ involves differentiation of the nonlinear expression for the phase speed only with respect to explicit dependence on the spatial coordinate $x$. That is, considering the non-homogeneity to arise solely through the function $\epsilon(\tilde{x})$, 

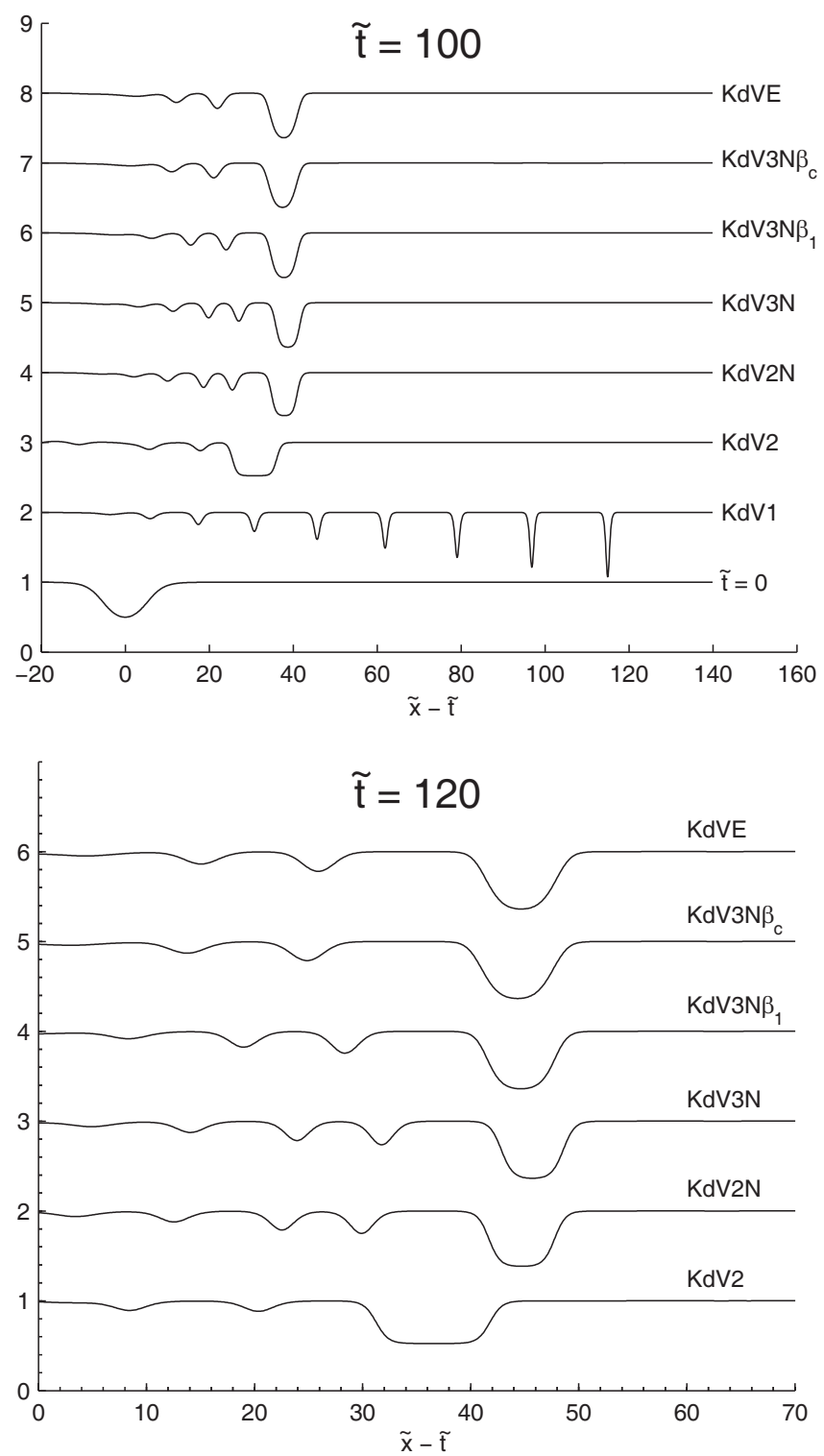

Fig. 9. Comparison of spatial wave forms at a fixed time as derived from various models using the same initial condition $(\epsilon=2 / 3)$.

and ignoring the implicit dependence through the amplitude function $\tilde{\zeta}(x, t)$. In this case, for example, the rarefaction term for the generalized equation (Eq. 26) becomes

$\frac{1}{2} \frac{\partial c}{\partial x}=\frac{1}{2}\left\{\tilde{c}_{E}(\tilde{\zeta}, \epsilon) \frac{d c_{0}}{d x}+c_{0} \frac{\partial \tilde{c}_{E}}{\partial \epsilon} \frac{d \epsilon}{d x}\right\}$.

In order to exhibit the effect of nonlinearity entering via this term, the initial value problem was solved using a depth variation where $\epsilon$ varied gradually (i.e., over a distance of roughly $200(=2 H)$ upper-layer depths) from its initial (upstream) value of $\epsilon_{1}=3 / 4$ to its shelf (downstream) value $\epsilon_{2}=1 / 3$ according to the relation (a)

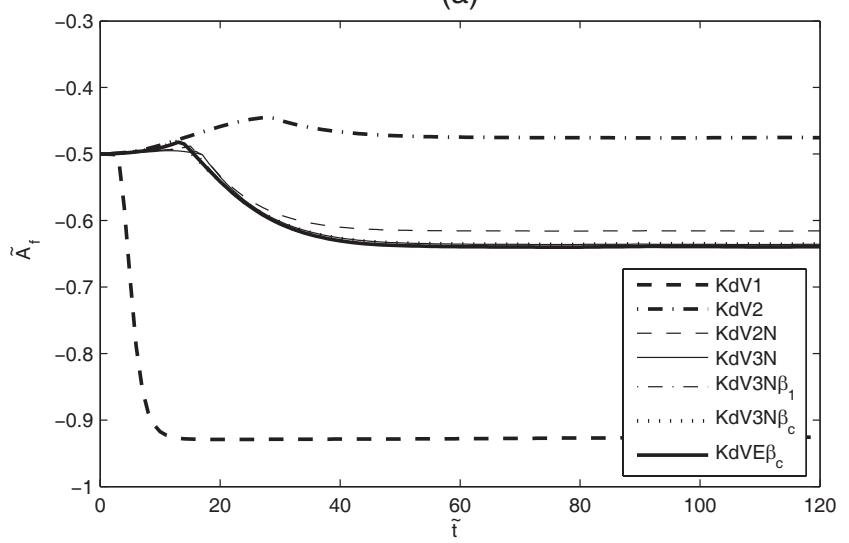

(b)

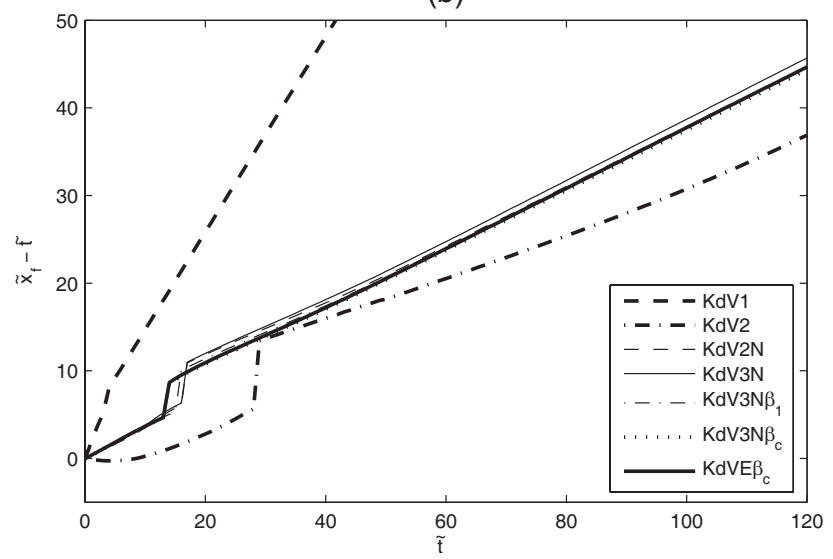

Fig. 10. Change in (a) leading wave front amplitude $\tilde{A}_{f}$ and (b) its position $\left(\tilde{x}_{f}-\tilde{t}\right)$ as a function of time $(\epsilon=2 / 3)$.

$\epsilon(\tilde{x})=\frac{\epsilon_{1}+\epsilon_{2}}{2}-\frac{\epsilon_{1}-\epsilon_{2}}{2} \tanh \left(\frac{\tilde{x}}{H}\right)$.

The initial condition in this simulation was the same as that used for the simulations presented in Fig. 9. Simulations were performed for both the weakly-nonlinear version KdV2 and for the strongly nonlinear version KdV2N. Waveforms obtained at several times are compared in Fig. 11. As expected, the KdV2N waveform travels faster, and has a narrower leading solitary wave since the limiting amplitude for $\mathrm{KdV} 2$ is smaller, resulting in a more extended and flattened trough. The waveforms appearing at a late time on the shelf are shown in Fig. 12.

It is apparent that a larger number of solitons are formed for KdV2N on the shelf region, although the trailing waveshelf has not yet fully evolved to a point when the solitary wave packet has separated from the dispersive tail. A curious structure, particularly in the KdV2 waveform, is the occurrence of two localized soliton pairs, as seemingly a 'phaselocked' state, following the lead wave. A similar sort of wave pair seems to be manifest at earlier times (e.g., $\tilde{t}=60)$ in the 


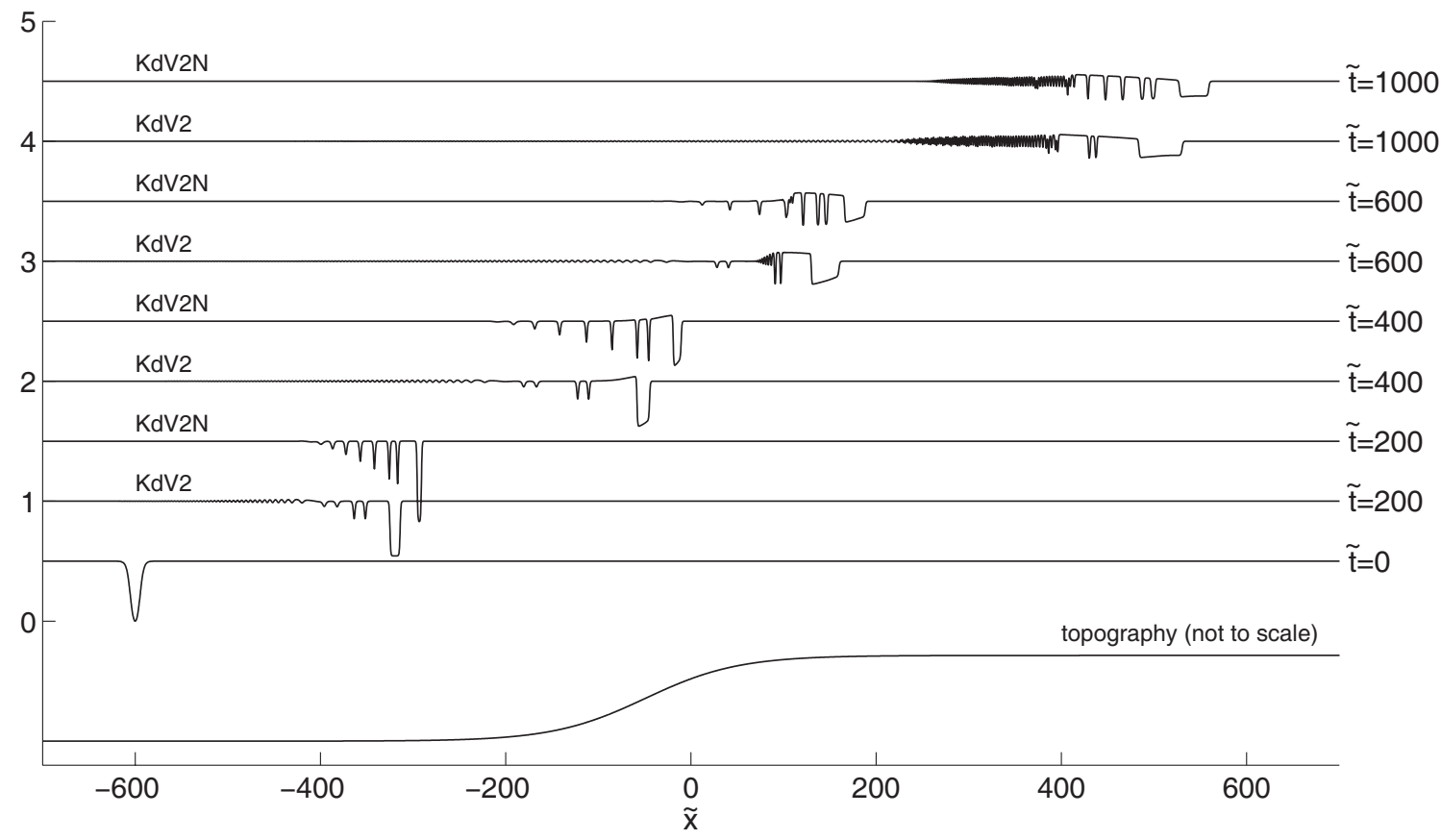

Fig. 11. Comparison of shoaling wave forms for $\mathrm{KdV} 2$ and $\mathrm{KdV} 2 \mathrm{~N}$ for evolution from a common initial condition.

KdV2N waveform, and has a remnant form even at $\tilde{t}=1800$. We speculate that the formation of this pair-like form occurs when the lead wave with near-limiting amplitude undergoes deformation while passing over the topographic variation. The individual entities in this pair-like form have (nearly) identical amplitudes and propagate with (nearly) fixed proximity to each other.

\section{Concluding remarks}

Several models have been proposed for the evolution of lowest-mode, internal wave disturbances based on the exact relation for the phase speed for nonlinear wave propagation along a single characteristic in the long wave (hydrostatic) limit. The proposed models have been examined for the purpose of providing a reliable, quantitative description of the evolutionary character of waves in a two-layer stratification with arbitrary amplitude. The need for models valid for strongly nonlinear evolution is readily seen when considering several documented cases. For example, waveforms with amplitudes $\left(\tilde{\zeta}_{1} \approx-0.8, \tilde{\zeta} \approx-0.32\right)$ in an environment with $\epsilon=0.59$ have been reported by Pingree and Mardell (1985); waveforms with $\left(\tilde{\zeta}_{1} \approx-2.1, \tilde{\zeta} \approx-0.32\right)$ in an environment with $\epsilon=0.85$ have been reported by Trevorrow (1998); and waveforms with $\left(\tilde{\zeta}_{1}=-4, \tilde{\zeta}=-0.4\right)$ in an environment with $\epsilon=0.91$ have been reported by Stanton and Ostrovsky (1998). In weakly nonlinear theory the asymptotic expansion presupposes that the characteristic amplitude parameter

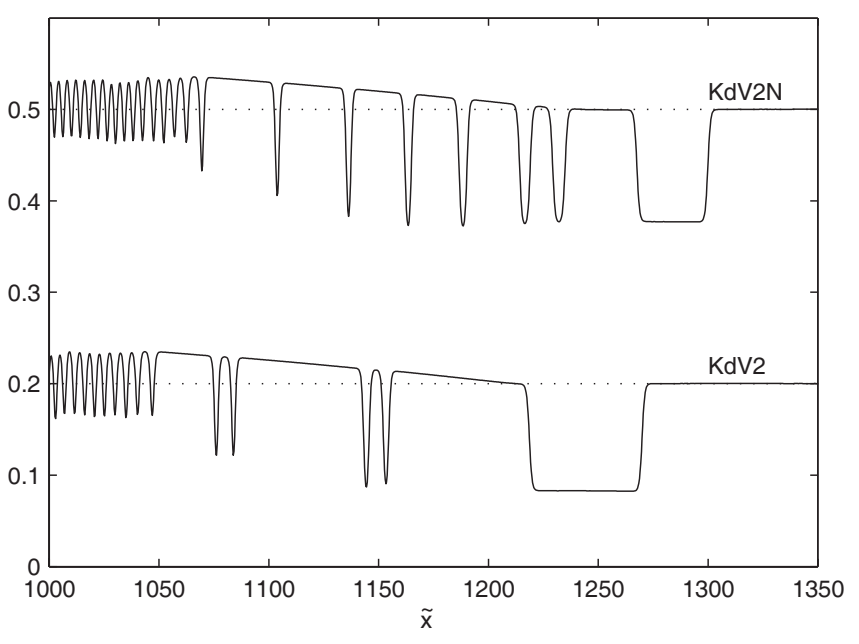

Fig. 12. Comparison of shoaling wave forms for $\mathrm{KdV} 2$ and $\mathrm{KdV} 2 \mathrm{~N}$ at $\tilde{t}=1800$.

$\left|\tilde{\zeta}_{1}\right| \ll 1$. In light of these observations, the need for reliable models for strongly nonlinear evolution is indeed obvious.

We reiterate one of the motivations for this work. Although the work of Choi and Camassa (1999) allows one to quite readily compute exact, equilibrium (stationary) solutions for isolated waves, there is a need for evolution models which allow rapid simulation for either predictive or interpretive purposes. It is our thesis that a "simple" two-layer model has significant advantages for these purposes. To this end, we include in Appendix B some calculations of the KdV2 model 
Table 4. Properties of solitary waves of $\operatorname{KdVE} \beta_{c}$ for environments having deep lower layers.

\begin{tabular}{|c|c|c|c|c|c|c|c|c|c|}
\hline \multirow[b]{2}{*}{$\tilde{\zeta}_{0}$} & \multicolumn{3}{|c|}{$\epsilon=4 / 5$} & \multicolumn{3}{|c|}{$\epsilon=7 / 8$} & \multicolumn{3}{|c|}{$\epsilon=9 / 10$} \\
\hline & $\tilde{X}_{i}$ & $\tilde{\zeta}_{i}$ & $\tilde{\zeta}_{\tilde{X}_{i}}$ & $\tilde{X}_{i}$ & $\tilde{\zeta}_{i}$ & $\tilde{\zeta}_{\tilde{X}_{i}}$ & $\tilde{X}_{i}$ & $\tilde{\zeta}_{i}$ & $\tilde{\zeta}_{\tilde{X}_{i}}$ \\
\hline-0.1 & 4.59 & -0.062 & 0.013 & 4.34 & -0.059 & 0.015 & 4.33 & -0.058 & 0.015 \\
\hline-0.2 & 4.49 & -0.117 & 0.029 & 4.56 & -0.111 & 0.031 & 4.69 & -0.108 & 0.031 \\
\hline-0.3 & 4.70 & -0.168 & 0.044 & 4.93 & -0.159 & 0.045 & 5.15 & -0.155 & 0.045 \\
\hline-0.4 & 5.03 & -0.217 & 0.057 & 5.34 & -0.205 & 0.058 & 5.62 & -0.199 & 0.057 \\
\hline-0.5 & 5.49 & -0.262 & 0.069 & 5.81 & -0.248 & 0.069 & 6.12 & -0.241 & 0.068 \\
\hline-0.6 & 6.22 & -0.302 & 0.078 & 6.42 & -0.288 & 0.078 & 6.74 & -0.280 & 0.077 \\
\hline-0.7 & 7.68 & -0.330 & 0.084 & 7.36 & -0.322 & 0.085 & 7.62 & -0.314 & 0.083 \\
\hline-0.8 & - & - & - & 9.53 & -0.343 & 0.090 & 9.39 & -0.338 & 0.088 \\
\hline
\end{tabular}

for a continuously stratified thermoclinic layer of finite thickness, and then provide suggested values of the layer depths to be used in an equivalent two-layer model.

Examination of Figs. 3 and 4 suggests that KdV2 would considerably underestimate the nonlinearity for all cases listed above. By contrast, the KdV2N evolutionary model could be expected to yield a respectably satisfactory degree of correspondence between model results and the level of $|\tilde{\zeta}|$ values associated with these observations. However, for cases where amplitudes are such that $|\tilde{\zeta}|>0.5-0.6, \mathrm{KdV} 3 \mathrm{~N}$ will be required to realize a reasonable fidelity between model results and measured data. Advantages of KdV2N include analytical expressions for both the form of a solitary wave and its eigenspeed, the same advantages associated with KdV1 and KdV2. Hence, wave properties can be readily computed and compared with laboratory or field data, and simple scaling laws for these waves are available. Furthermore, KdV2N provides a quite respectable approximation for the limiting amplitude, particularly in comparison to $\mathrm{KdV} 2$, as seen in Table 2. Of course, an analytical expression for solitary wave solutions of KdV3N is certainly expected to be accessible as well, although preliminary efforts on our part have not yet yielded success in this venture. Hence, the argument in favor of KdV2N over KdV3N on the basis of possessing a solitary wave solution, with its specific scaling relationships, is not entirely compelling.

Comparing data for solitary wave profiles for KdV2N and KdV3N contained in Table 3, it is clear that the difference between the indicated values of the width of the wave profile only becomes significant as the limiting amplitude is approached. On the other hand, comparison of profile widths for $\operatorname{KdV} 3 \mathrm{~N}$ with those for $\operatorname{KdV} 3 \mathrm{~N} \beta_{1}$ and $\operatorname{KdV} 3 \mathrm{~N} \beta_{c}$, it is seen that nonlinear dispersive effects serve to increase the wave width significantly, with the exception being when wave amplitudes are near their limiting value for a specific model. With this effect of nonlinear dispersion in view, it is worth noting that, although no data have been included herein, one could readily compute corresponding properties for such cases as $\mathrm{KdV} 2 \mathrm{~N} \beta_{1}$ and $\mathrm{KdV} 2 \mathrm{~N} \beta_{c}$. Furthermore, since physical environments with relatively deep lower layers (i.e., values of $\epsilon$ approaching unity) are not uncommon, data pertaining to $\mathrm{KdVE} \beta_{c}$ for several depth ratios are presented in Table 4.

\section{Appendix A}

The eigenvalue relation for case $\operatorname{KdV} 3 \mathrm{~N} \beta_{c}$ is, after carrying through the evaluation of the integral terms in Eq. (34),

$$
\tilde{v}=\tilde{V}-1=-\frac{1}{J_{V}}\left\{\frac{\tilde{\mu}_{1}}{2} J_{\mu_{1}}+\frac{\tilde{\mu}_{2}}{3} J_{\mu_{2}}+\frac{\tilde{\mu}_{3}}{4} J_{\mu_{3}}\right\} .
$$

The various $J_{k}$ terms in this relation are defined as

$$
\begin{aligned}
J_{V}= & -\frac{d_{m}}{2}\left\{(1-P) \ln \left(\frac{1+S}{1-P}\right)\right. \\
& \left.+(1+P) \ln \left(\frac{1-S}{1+P}\right)\right\} ; \\
J_{\mu_{1}}= & -d_{m}^{2}(P+S)+\frac{d_{m}^{2}}{2}\left\{(1-P)^{2} \ln \left(\frac{1+S}{1-P}\right)\right. \\
& \left.-(1+P)^{2} \ln \left(\frac{1-S}{1+P}\right)\right\} ; \\
J_{\mu_{2}=} & -\frac{d_{m}^{3}}{2}\left\{\left(S^{2}-P^{2}\right)+6 d_{m}(S+P)\right. \\
& \left.+(1-P)^{3} \ln \left(\frac{1+S}{1-P}\right)+(1+P)^{3} \ln \left(\frac{1-S}{1+P}\right)\right\} ; \\
J_{\mu_{3}=} & -d_{m}^{4}\left\{\left(1+6 d_{m}^{2}\right)(P+S)+2 d_{m}\left(S^{2}-P^{2}\right)\right. \\
& \left.+\frac{1}{3}\left(P^{3}+S^{3}\right)\right\}+\frac{d_{m}^{4}}{2}\left\{(1-P)^{4} \ln \left(\frac{1+S}{1-P}\right)\right. \\
& \left.-(1+P)^{4} \ln \left(\frac{1-S}{1+P}\right)\right\} .
\end{aligned}
$$

www.nonlin-processes-geophys.net/14/31/2007/ 
In writing these expressions we have employed the definition

$$
P=\frac{\tilde{\zeta}_{D m}}{d_{m}} ; \quad S=\frac{\tilde{\zeta}-\tilde{\zeta}_{D m}}{d_{m}} .
$$

In a corresponding manner, the relevant $J_{k}$ terms for $\operatorname{KdV} 3 \mathrm{~N} \beta_{1}$ are, upon using the definition $S_{1}=\tilde{\zeta}_{1}+\epsilon$,

$$
\begin{aligned}
J_{V}= & -\frac{1}{2}\left\{(1-\epsilon) \ln \left(\frac{1+S_{1}}{1+\epsilon}\right)\right. \\
& \left.+(1+\epsilon) \ln \left(\frac{1-S_{1}}{1-\epsilon}\right)\right\} ; \\
J_{\mu_{1}}= & -\tilde{\zeta}+\frac{1}{2}\left\{(1+\epsilon)^{2} \ln \left(\frac{1+S_{1}}{1+\epsilon}\right)\right. \\
& \left.-(1-\epsilon)^{2}\left(\frac{1-S_{1}}{1-\epsilon}\right)\right\} ; \\
J_{\mu_{2}=} & 3 \epsilon \tilde{\zeta}-\frac{1}{2}\left(S_{1}^{2}-\epsilon^{2}\right)-\frac{1}{2}\left\{(1+\epsilon)^{3} \ln \left(\frac{1+S_{1}}{1+\epsilon}\right)\right. \\
& \left.+(1-\epsilon)^{3} \ln \left(\frac{1-S_{1}}{1-\epsilon}\right)\right\} ; \\
J_{\mu_{3}=} & -\left(1+6 \epsilon^{2}\right) \tilde{\zeta}+2 \epsilon\left(S_{1}^{2}-\epsilon^{2}\right) \\
& -\frac{1}{3}\left(S_{1}^{3}-\epsilon^{3}\right)+\frac{1}{2}\left\{(1+\epsilon)^{4} \ln \left(\frac{1+S_{1}}{1+\epsilon}\right)\right. \\
& \left.-(1-\epsilon)^{4} \ln \left(\frac{1-S_{1}}{1-\epsilon}\right)\right\} .
\end{aligned}
$$

\section{Appendix B}

We present calculations aimed at guiding the formation of an equivalent two-layer model to characterize long wave propagation in a wave guide which contains a thermoclinic layer of finite thickness. For this purpose we consider an environmental model where a single thermoclinic layer with constant Brunt-Väisälä frequency having thickness $h_{m}$ is positioned in a wave guide such that the distance from the upper surface to the mid-depth of the stratified layer is $h_{10}$. The total depth of the wave guide is $h_{10}+h_{20}, h_{20}$ being the distance from the middle of the thermocline to the bottom of the wave guide. The goal is to specify the equivalent upper-layer (mixed layer) depth $h_{\text {equiv }}=h_{1}$ in a two-layer environmental model with total fluid depth $h_{1}+h_{2}=h_{10}+h_{20}$ for different thicknesses $h_{m}$ of the thermoclinic layer.

Several different criteria for defining an equivalent twolayer model can be proposed. To establish conditions for an equivalent model, the value of the reduced gravity $\tilde{g}=g\left(\rho_{1}-\rho_{2}\right) / \rho_{1}$ must be specified. We take $\rho_{1}$ and $\rho_{2}$ to be the epilimnion and hypolimnion densities in the continuously-stratified environment. Thus, within the Boussinesq approximation, the value of $\tilde{g}$ is equal to the integral of the Brunt-Väisälä frequency across the depth $h_{m}$ of the stratified layer. With $\tilde{g}$ fixed, one further condition must be prescribed to fix the remaining free parameter - the equivalent upper-layer depth $h_{\text {equiv }}$ (equal to $h_{1}$ in the text of this

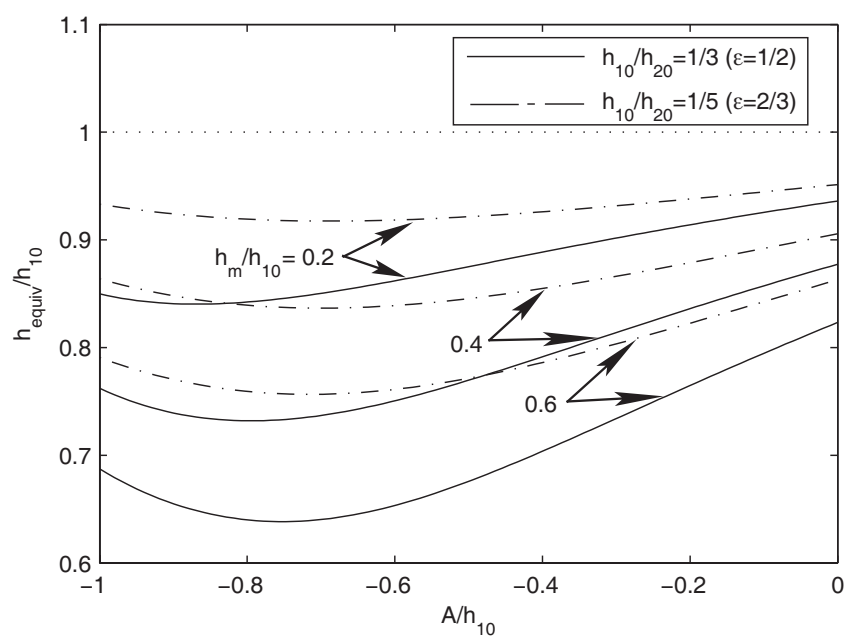

Fig. B1. Equivalent upper layer depth based on matching phase speed of solitary wave as a function of wave amplitude.

paper). We present calculations here based on two different options for this purpose, and then compare the consequent values of $h_{\text {equiv. }}$. First, we constrain the speed of lowestmode, isolated (solitary) wave features in the continuouslystratified case to correspond with those in an equivalent twolayer model. This criterion forces a correspondence between the location of waves relative to their source, and also phase relationships between different waves in a packet. Second, we choose to match the peak isopycnal displacement (wave amplitude) in the continuously-stratified model with the interface displacement in the equivalent two-layer model.

It is immediately clear that each of these bases for equivalence depend on the wave amplitude. In order to establish equivalence, therefore, it seems that comparable approximations for nonlinear and dispersive effects should be employed for each environmental model. However, since a fully-nonlinear representation of the nonlinear phase speed comparable to Eq. (6) is not available for the continuouslystratified case, we choose to use the KdV2 description of wave evolution for both environments. For the two-layer model we employ the relations given by Eqs. (1-5) in the text, and for the continuously-stratified wave-guide we use the KdV2 description as presented by Grimshaw (2002).

Results for the upper-layer depth for the equivalent twolayer model based on the criterion of matching the nonlinear phase speeds of equal-amplitude solitary waves are given in Fig. B1 for two different cases, depth ratios corresponding to $h_{10} / h_{20}=1 / 3(\epsilon=1 / 2)$ and $h_{10} / h_{20}=1 / 5(\epsilon=2 / 3)$. Solitary waves in environments with these depth ratios are waves are of depression (alt., waves of elevation with respect to the shallower upper layer). Values of $h_{\text {equiv }}$ and $A$ presented in Fig. B1 are scaled by $h_{10}$, the depth of the mid-level of the thermoclinic layer. One observes that the equivalent upper-layer depth is less than the mid-level depth $h_{10}$, and 


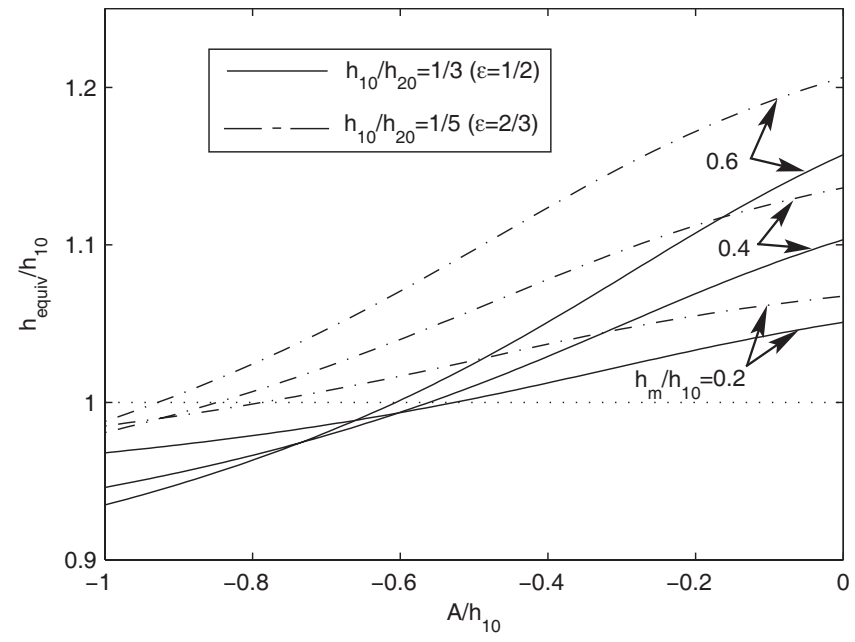

Fig. B2. Equivalent upper layer depth based on matching peak isopycnal displacement as a function of wave amplitude.

the reduction in equivalent upper-layer depth increases as the thickness $h_{m}$ of the thermoclinic layer increases. Further, the variation of $h_{\text {equiv }}$ with wave amplitude is much weaker when the layer depths are less disparate. In fact, in the deeper case with $h_{10} / h_{20}=1 / 5$, the results show that the $h_{\text {equiv }}>h_{10}$ when the wave amplitude becomes large (i.e., larger negative values). Of course, if the results shown in Fig. 3 for the two-layer model are also indicative of corresponding results for the continuously-stratified case, the utility of the KdV2 approximation should properly be restricted to amplitudes $\tilde{A}=|A / h|<0.3$.

Figure B2 presents results for the equivalent two-layer depth $h_{\text {equiv }}$ based on the alternate criterion of matching the peak isopycnal displacement. To realize corresponding isopycnal displacements, the value of $h_{\text {equiv }}$ is found, in contrast to results shown in Fig. B1, to be greater than the mid-thermocline depth $h_{10}$. Furthermore, the value of $h_{\text {equiv }}$ based on this criterion exhibits quite sensitive amplitude dependence, with $h_{\text {equiv }}$ decreasing toward the mid-thermocline depth as the wave amplitude increases.

This brief consideration of issues underlying the selection of an equivalent two-layer model shows clearly that some physical effects must be compromised in the use of a simplified environmental model. In this connection, we prefer to base the equivalent model on the first criterion. That is, we prefer that wave arrival times and phase information be replicated as accurately as possible. Employing this criterion, values of $h_{\text {equiv }}$ have been computed for several choices of the parameters eps and $h_{m} / h_{10}$. The computed values of $h_{\text {equiv }}$, having the form shown in Fig. B1, were then averaged over that range of wave amplitudes satisfying the condition $0.9 \leq\left|c_{\mathrm{KdV} 2} / c_{E}\right| \leq 1.0$ for the two-layer model. Results of this calculation are summarized in Table B1. We suggest that the values of $h_{\text {equiv }}$ presented in Table B1 should
Table B1. Equivalent upper layer depth for different thickness of thermoclinic layer.

\begin{tabular}{cc|ccccc}
\hline & & \multicolumn{5}{|c}{$h_{m} / h_{10}$} \\
$h_{10} / h_{20}$ & $\epsilon$ & 0.2 & 0.4 & 0.6 & 0.8 & 1.0 \\
\hline $1 / 3$ & $1 / 2$ & 0.88 & 0.79 & 0.71 & 0.63 & 0.56 \\
$1 / 5$ & $2 / 3$ & 0.93 & 0.86 & 0.79 & 0.72 & 0.66 \\
$1 / 7$ & $3 / 4$ & 0.94 & 0.88 & 0.81 & 0.75 & 0.69 \\
$1 / 9$ & $4 / 5$ & 0.94 & 0.88 & 0.82 & 0.76 & 0.70 \\
$1 / 11$ & $5 / 6$ & 0.94 & 0.89 & 0.83 & 0.77 & 0.71 \\
$1 / 13$ & $6 / 7$ & 0.94 & 0.89 & 0.83 & 0.77 & 0.72 \\
\hline
\end{tabular}

provide a useful guide for defining quantitatively equivalent two-layer models for natural environments containing single, prominent thermocline having finite thickness.

Edited by: A. Osborne

Reviewed by: two referees

\section{References}

Amick, C. and Turner, R.: A global theory of internal waves in twofluid systems., Trans. Am. Math. Soc., 298, 431-481, 1986.

Antenucci, J., Imberger, J., and Saggio, A.: Seasonal evolution of the basin-scale internal wave field in a large stratified lake., Limnol. Oceanogr., 45, 1621-1638, 2000.

Apel, J., Holbrook, J., Liu, A., and Tsai, J.: The Sulu Sea internal soliton experiment., J. Phys. Oceanogr., 15, 1625-1651, 1985.

Benjamin, T.: Internal waves of finite amplitude and permanent form., J. Fluid Mech., 25, 241-270, 1966.

Bogucki, D. and Redekopp, L.: A mechanism for sediment resuspension by internal solitary waves., Geophys. Res. Lett., 26, 1317-1320, 1999.

Bogucki, D., Dickey, T., and Redekopp, L.: Sediment resuspension and mixing by resonantly generated internal solitary waves., J. Phys. Oceanogr., 27, 1181-1196, 1997.

Bogucki, D., Redekopp, L., and Barth, J.: Internal solitary waves in the Coastal Mixing and Optics 1996 experiment: Multimodal structure and resuspension, J. Geophys. Res., 110, C02024, doi:10.1029/2003JC002253, 2005.

Choi, W. and Camassa, R.: Fully nonlinear internal waves in a twofluid system., J. Fluid Mech., 396, 1-36, 1999.

Djordjevic, V. and Redekopp, L.: The fission and disintegration of internal solitary waves moving over two-dimensional topography., J. Phys. Oceanogr., 8, 1016-1024, 1978.

Duda, T., Lynch, J., Irish, J., Reardsley, R., Ramp, S., Chui, C., Tang, T., and Yang, Y.: Internal tide and nonlinear internal wave behavior at the continental slope in the northern South China Sea., IEEE J. Oceanic Eng., 29, 1105-1130, 2004.

Evans, W. and Ford, M.: An integral equation approach to internal (2-layer) solitary waves., Phys. Fluids, 8, 2032-2047, 1996.

Grimshaw, R.: Internal solitary waves, chap. 1, pp. 1-27, Kluwer Academic, Boston, 2002. 
Helfrich, K. and Melville, W.: Long nonlinear internal waves., Annu. Rev. Fluid Mech., 38, 395-425, 2006.

Holloway, P. and Pelinovsky, E.: Internal solitary waves, chap. 2, pp. 29-60, Kluwer Academic, Boston, 2002.

Lamb, K. and Wan, B.: Conjugate flows and flat solitary waves for a continuously stratified fluid., Phys. Fluids, 10, 2061-2079, 1998.

Osborne, A. and Burch, T.: Internal solitons in the Andaman Sea., Science, 208, 451-460, 1980.

Ostrovsky, L. and Grue, J.: Evolution equations for strongly nonlinear internal waves., Phys. Fluids, 15, 2934-2948, 2003.

Ostrovsky, L. and Stepanyants, Y. A.: Do internal solitons exist in the ocean?, Rev. Geophys., 27, 293-310, 1989.

Ostrovsky, L. and Stepanyants, Y. A.: Internal solitons in laboratory experiments: Comparison with theoretical models, Chaos, 15, 037 111, doi:10.1063/1.2107087, 2005.

Pingree, R. and Mardell, G.: Solitary internal waves in the Celtic Sea., Prog. Oceanogr., 14, 431-441, 1985.

Scotti, A. and Pineda, J.: Observation of very large and steep internal waves of elevation near the Massachusssetts coast., Geophys. Res. Lett., 31, L22 307, doi:1029/2004GL021052, 2004.
Slunyaev, A., Pelinovsky, E., Poloukhina, O., and Gavrilyuk, S.: The Gardner equation as the model for long internal waves., in: Topical Problems of Nonlinear Wave Physics, Proc. Int'1. Symp., pp. 368-369, Inst. of Appl. Phys., RAS, Nizhny Novgorod, 2003.

Stanton, T. and Ostrovsky, L.: Observations of highly nonlinear internal solitons over the continental shelf., Geophys. Res. Lett., 25, 2695-2698, doi:10.1029/98GL01772, 1998.

Stastna, M. and Lamb, K.: Vortex shedding and sediment resuspention associated with the interaction of an internal solitary wave and the bottom boundary layer., Geophys. Res. Lett., 29(11), 1512, doi:10.1029/2001GL014070, 2002.

Trevorrow, M.: Observations of internal solitary waves near the Oregon coast with an inverted echo sounder., J. Geophys. Res. - Oceans, 1041, 7671-7680, 1998.

Turner, R. and Vanden-Broeck, J.: Broadening of interfacial solitary waves., Phys. Fluids, 31, 2486-2490, 1988. 